\title{
Adaptive time step algorithms for the simulation of marine ecosystem models using the transport matrix method implementation Metos3D (v0.5.0)
}

\author{
Markus Pfeil ${ }^{1}$ and Thomas Slawig ${ }^{1}$ \\ ${ }^{1}$ KMS - Centre for Interdisciplinary Marine Science, Dept. of Computer Science, Kiel University, 24098 Kiel, Germany \\ ( $\{\mathrm{mpf}, \mathrm{ts}\} @$ informatik.uni-kiel.de).
}

Correspondence: Markus Pfeil (mpf@informatik.uni-kiel.de)

\begin{abstract}
The reduction of the computational effort is desirable for the simulation of marine ecosystem models. Using a marine ecosystem model, the assessment and the validation of annual periodic solutions (i.e., steady annual cycles) against observational data are crucial to identify biogeochemical processes, which, for example, influence the global carbon cycle. For marine ecosystem models, the transport matrix method (TMM) already lowers the runtime of the simulation significantly enables the application of larger time steps straightforwardly. However, the selection of an appropriate time step is a challenging compromise between accuracy and shortening the runtime. Using an automatic time step adjustment during the computation of a steady annual cycle with the TMM, we present in this paper different algorithms applying either an adaptive step size control or decreasing time steps in order to use the time step always as large as possible without any manual selection. For these methods and a variety of marine ecosystem models of different complexity, the accuracy of the computed steady annual cycle achieved the same accuracy as solutions obtained with a fixed time step. Depending on the complexity of the marine ecosystem model, the application of the methods shortened the runtime significantly. Due to the certain overhead of the adaptive method, the computational effort may be higher in special cases using the adaptive step size control. The presented methods represent computational efficient methods for the simulation of marine ecosystem models using the TMM but without any manual selection of the time step.
\end{abstract}

\section{Introduction}

In climate research, marine ecosystem models are important for the assessment of the role of the marine ecosystem in climate change. Diverse biogeochemical processes influence the changes of the marine ecosystem. As part of the global carbon cycle, one aim, for example, is to identify biogeochemical processes that affect the $\mathrm{CO}_{2}$ uptake and storage of the ocean. Marine ecosystem models take the interplay of the ocean circulation and the biogeochemical processes into account and, therefore, consists of a global circulation model coupled to a biogeochemical model (cf. Fasham, 2003; Sarmiento and Gruber, 2006). The equations and variables describing the ocean dynamics (i.e., the physical processes) are well known. In contrast, many different biogeochemical models exist differing in the complexity by the number of state variables and parametrizations, because there is, in general, no set of equations and variables to describe the biogeochemical processes (see e.g., Kriest et al., 2010). Accordingly, 
https://doi.org/10.5194/gmd-2021-392

Preprint. Discussion started: 7 January 2022

(c) Author(s) 2022. CC BY 4.0 License.

(c) (i)

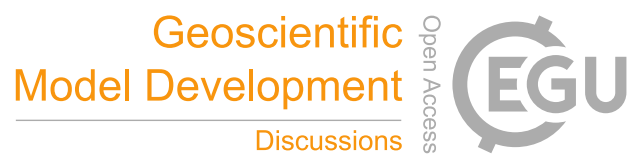

a validation of the different biogeochemical models is necessary including the assessment of the ability of the model output to reproduce observational data (cf. Fennel et al., 2001; Schartau and Oschlies, 2003). This assessment involves both a parameter optimization and a discussion of the simulation results.

Simulation runs with marine ecosystem models are computationally expensive. In three spatial dimensions, the simultaneous computation of the ocean circulation and the biogeochemical model already results in a high computational effort for a single evaluation of a marine ecosystem model (Oschlies, 2006). Thus, the computational effort of the computation of a steady annual cycle is enormous because it requires a long-time integration over several millennia (cf. Bernsen et al., 2008; Bryan, 1984; Danabasoglu et al., 1996; Wunsch and Heimbach, 2008; Siberlin and Wunsch, 2011). For applications requiring a high number of model evaluations (such as parameter sensitivity, uncertainty or identification studies (e.g. Kriest, 2017; Kriest et al., 2017)), the high computational effort becomes even more obstructive.

Several strategies focus on the reduction of the computational effort to compute a steady annual cycle of a marine ecosystem model (e.g., Bryan, 1984; Danabasoglu et al., 1996; Wang, 2001; Khatiwala et al., 2005). Using domain decomposition methods, parallelization, firstly, lowers the computational effort. Instead of using a fully coupled simulation (also called online simulation), the offline model, secondly, reduces the computational effort. More specifically, an offline model considers only the one-way coupling of the influence of the ocean circulation on the biogeochemical model. Due to the neglect of the impact of the biogeochemical model on the circulation, an offline model can use pre-computed data of the ocean circulation. Khatiwala et al. (2005), thirdly, introduced with the transport matrix method (TMM) a method reducing the computational effort with a tolerable loss of accuracy (see also Khatiwala, 2007). The TMM approximates the computation of the global ocean circulation by matrix-vector multiplications and, hence, decouples the evaluation of the biogeochemical model from the ocean circulation. Moreover, Khatiwala (2008) replaced the long-time integration in the TMM by the use of Newton's method. Lastly, the computation of steady annual cycles using graphics processing units shortens the computational time (Siewertsen et al., 2013). These methods are only four examples of the strategies to reduce the computational effort.

The used time step affects both the computational effort and the accuracy of the steady annual cycle computation. The application of larger time steps obviously reduces the computational effort whereas the accuracy of the steady annual cycle approximation decreases with a larger time step. In many cases and, specifically, for less complex models, approximations of the same steady annual cycles as the ones obtained with the standard time step can be computed with bigger ones (Pfeil and Slawig, 2021a). The obtained steady annual cycles were the same as for small time steps, but achieved with slightly reduced accuracy. In fact, some studies using the TMM for parameter optimization, where the runtime is a crucial point, have already used larger time steps (e.g., Prieß et al., 2013; Kriest et al., 2017). However, the selection of a suitable time step is a challenging task, also in the TMM. The time step should be chosen as large as possible to keep the computational effort low, but the accuracy of the approximation cannot be neglected. In this paper, we present methods adjusting automatically the time steps during the computation of the steady annual cycle. One approach is an adaptive step size control that, depending on an error estimation, adjusts the time step during the simulation. Here, we studied two methods ignoring and avoiding negative tracer concentrations during the step size control, respectively. The third method starts with a possible large time step and uses it as long as possible before the time step is decreased. For this purpose, the method checks the progress of the steady annual cycle computation 
using the current time step. In particular, all methods use the largest possible time step to shorten the runtime of the simulation of marine ecosystem models.

This paper is structured as follows: Sect. 2 contains a description of marine ecosystem models including the computation of steady annual cycles. In Sects. 3 and 4, we introduce the three methods used to compute a steady annual cycle with an automatic time step adjustment. Numerical results for the steady annual cycle computation are presented in Sect. 5. The paper closes with a summary and conclusions (Sect. 6).

\section{Model description}

A marine ecosystem model represents the interaction between the ocean circulation, the ocean biota and marine biogeochemical cycles. This involves modeling the marine ecosystem by a given number of ecosystem species (or biogeochemical tracers), which are substances in the ocean water and subject to chemical or biochemical reactions. Due to the fully coupling of the ocean circulation with the tracers, in which the circulation influences the tracer concentrations and, vice versa, the tracer concentrations affect the circulation, the simulation of a fully coupled model (online model) is computationally expensive and limited to single model evaluations. Restricting this coupling to the influence of the ocean circulation on the tracer concentrations (i.e., an offline model using passive tracers that do not affect the ocean circulation) reduces the computational effort. In particular, this one-way coupling enables the application of a pre-computed ocean circulation for the simulation.

\subsection{Model equations for marine ecosystems}

A system of partial differential equations describes the marine ecosystem model. The number of modeled tracers defines the complexity of the marine ecosystem model and, hence, the size of the system of differential equations. For $n_{y} \in \mathbb{N}$ tracers on a spatial domain $\Omega \subset \mathbb{R}^{3}$ (i.e., the ocean) and a time interval $[0,1]$ (i.e., one model year), we consider, hereinafter, marine ecosystem models using an offline model. With the function $y_{i}: \Omega \times[0,1] \rightarrow \mathbb{R}, i \in\left\{1, \ldots, n_{y}\right\}$, of the tracer concentrations for the single tracer $y_{i}$ and the vector $\boldsymbol{y}:=\left(y_{i}\right)_{i=1}^{n_{y}}$ of all tracers, the system of parabolic partial differential equations

$80 \frac{\partial y_{i}}{\partial t}(x, t)+(D(x, t)+A(x, t)) y_{i}(x, t)=q_{i}(x, t, \boldsymbol{y}, \boldsymbol{u}), \quad x \in \Omega, t \in[0,1]$,

$$
\frac{\partial y_{i}}{\partial n}(x, t)=0, \quad x \in \partial \Omega, t \in[0,1]
$$

$i \in\left\{1, \ldots, n_{y}\right\}$, represents the tracer transport of a marine ecosystem model. The homogeneous Neumann boundary conditions (2) including the normal derivative models no fluxes on the boundary.

The advection and diffusion, coming from the ocean circulation, determines the tracer transport in marine water. For a given velocity field $v: \Omega \times[0,1] \rightarrow \mathbb{R}^{3}$, the linear operator $A: \Omega \times[0,1] \rightarrow \mathbb{R}$ models the advection as

$A(x, t) y_{i}(x, t):=\operatorname{div}\left(v(x, t) y_{i}(x, t)\right), \quad x \in \Omega, t \in[0,1]$,

for $i \in\left\{1, \ldots, n_{y}\right\}$. The diffusion $D: \Omega \times[0,1] \rightarrow \mathbb{R}$ used as model of the turbulent effects of the ocean circulation neglects the molecular diffusion of the tracers themselves since this is known to be much smaller in relation to the diffusion caused by 
turbulence. As a result of the quite different scales in horizontal and vertical direction, the diffusion operator is split into a sum $D=D_{h}+D_{v}$ of a horizontal and vertical part in order to treat the vertical part in the time integration implicitly. The diffusion is split into horizontal and vertical part as

$D_{h}(x, t) y_{i}(x, t):=-\operatorname{div}_{h}\left(\kappa_{h}(x, t) \nabla_{h} y_{i}(x, t)\right), \quad x \in \Omega, t \in[0,1]$,

$D_{v}(x, t) y_{i}(x, t):=-\frac{\partial}{\partial z}\left(\kappa_{v}(x, t) \frac{\partial y_{i}}{\partial z}(x, t)\right), \quad x \in \Omega, t \in[0,1]$,

for $i \in\left\{1, \ldots, n_{y}\right\}$. Here, $\operatorname{div}_{h}$ and $\nabla_{h}$ denote the horizontal divergence and gradient, respectively, and $z$ the vertical coordinate. The diffusion coefficient fields $\kappa_{h}, \kappa_{v}: \Omega \times[0,1] \rightarrow \mathbb{R}$ are the same for all tracers due to the fact that the molecular diffusion is generally assumed to be smaller than the diffusion induced by the turbulence of the ocean circulation.

The biogeochemical model contains the biogeochemical processes within the marine ecosystem. The interplay of the biogeochemical model with the effects of the ocean circulation (i.e., the whole system (1) to (6)), on the other hand, is called marine ecosystem model. For the tracer $y_{i}, i \in\left\{1, \ldots, n_{y}\right\}$, the, in general, nonlinear function $q_{i}: \Omega \times[0,1] \rightarrow \mathbb{R},(x, t) \mapsto q_{i}(x, t, \boldsymbol{y}, \boldsymbol{u})$ describes the biogeochemical processes for this tracer. In particular, this nonlinear function $q_{i}$ includes firstly the influence of the variability of the solar radiation at space and time, secondly the coupling of this tracer to the other species and thirdly $n_{u} \in \mathbb{N}$ model parameters $\boldsymbol{u} \in \mathbb{R}^{n_{u}}$ controlling, for example, growth, loss and mortality rates or sinking speed of this tracer. Altogether, the biogeochemical model $\boldsymbol{q}=\left(q_{i}\right)_{i=1}^{n_{y}}$ summarizes the biogeochemical processes of all tracers.

An annual periodic solution of the marine ecosystem (i.e., a steady annual cycle) satisfies in addition to (1) and (2)

$y_{i}(x, 0)=y_{i}(x, 1), \quad x \in \Omega$,

for $i \in\left\{1, \ldots, n_{y}\right\}$. For this purpose, we assume that the operators $D, A$ and the functions $q_{i}$ are also annually periodic in time.

\subsection{Semi-discrete setting}

For the adaptive step-size control, we used a semi-discrete setting where the computational domain is already discretized in space, but time is kept continuous. Then the above equations read

$110 \frac{\partial \boldsymbol{y}_{i}}{\partial t}(t)+(\mathbf{D}(t)+\mathbf{A}(t)) \boldsymbol{y}_{i}(t)=\boldsymbol{q}_{i}(t, \boldsymbol{y}(t), \boldsymbol{u}), \quad t \in[0,1]$,

with initial value $\boldsymbol{y}_{i}(0)=\boldsymbol{y}_{i}^{0}$.

\subsection{Biogeochemical models}

The biogeochemical models, used in this paper, differed in the number of ecosystem species. Kriest et al. (2010) introduced a hierarchy of five biogeochemical models with an increasing complexity starting with a simple model including only one tracer up to a model with five tracers. In addition to this hierarchy, we applied the biogeochemical model of Dutkiewicz et al. (2005). In the following, we briefly describe the biogeochemical models and refer to Kriest et al. (2010); Piwonski and Slawig (2016a); Dutkiewicz et al. (2005) for a detailed description of the modeled processes and model equations. Table 1 summarizes the model parameters of the different biogeochemical models. 
Table 1. Model parameters of the biogeochemical models.

\begin{tabular}{|c|c|c|}
\hline Parameter & Description & Unit \\
\hline$k_{w}$ & Attenuation coefficient of water & $\mathrm{m}^{-1}$ \\
\hline$k_{c}$ & Attenuation coefficient of phytoplankton & $\left(\mathrm{mmolP} \mathrm{m}{ }^{-3}\right)^{-1} \mathrm{~m}^{-1}$ \\
\hline$\mu_{P}$ & Maximum growth rate & $\mathrm{d}^{-1}$ \\
\hline$\mu_{Z}$ & Maximum grazing rate & $\mathrm{d}^{-1}$ \\
\hline$K_{N}$ & Half saturation constant for $\mathrm{PO}_{4}$ uptake & $\mathrm{mmol} \mathrm{P} \mathrm{m}{ }^{-3}$ \\
\hline$K_{P}$ & Half saturation constant for grazing & $\mathrm{mmol} \mathrm{P} \mathrm{m}{ }^{-3}$ \\
\hline$K_{I}$ & Light intensity compensation & $\mathrm{W} \mathrm{m}^{-2}$ \\
\hline$\sigma_{Z}$ & Fraction of production remaining in $\mathrm{Z}$ & 1 \\
\hline$\sigma_{\mathrm{DOP}}$ & Fraction of phytoplankton and zooplankton losses assigned to DOP & 1 \\
\hline$\lambda_{P}$ & Linear phytoplankton loss rate & $\mathrm{d}^{-1}$ \\
\hline$\kappa_{P}$ & Quadratic phytoplankton loss rate & $\left(\mathrm{mmolP} \mathrm{m}{ }^{-3}\right)^{-1} \mathrm{~d}^{-1}$ \\
\hline$\lambda_{Z}$ & Linear zooplankton loss rate & $\mathrm{d}^{-1}$ \\
\hline$\kappa_{Z}$ & Quadratic zooplankton loss rate & $\left(\mathrm{mmolP} \mathrm{m}{ }^{-3}\right)^{-1} \mathrm{~d}^{-1}$ \\
\hline$k_{c}$ & Attenuation coefficient of phytoplankton & $\left(\operatorname{mmol~P~m}{ }^{-3}\right)^{-1} \mathrm{~d}^{-1}$ \\
\hline$\lambda_{P}^{\prime}$ & Phytoplankton mortality rate & $\mathrm{d}^{-1}$ \\
\hline$\lambda_{Z}^{\prime}$ & Zooplankton mortality rate & $\mathrm{d}^{-1}$ \\
\hline$\lambda_{D}^{\prime}$ & Degradation rate & $\mathrm{d}^{-1}$ \\
\hline$\lambda_{\mathrm{DOP}}^{\prime}$ & Decay rate & $\mathrm{yr}^{-1}$ \\
\hline$b$ & Implicit representation of sinking speed & 1 \\
\hline$a_{D}$ & Increase of sinking speed with depth & $d^{-1}$ \\
\hline$b_{D}$ & Initial sinking speed & $\mathrm{md}^{-1}$ \\
\hline
\end{tabular}

Especially, the biological production depends on the available light. The light intensity decreases with the depth wherefore the ocean is divided into two layers, a euphotic (sun lit) layer of about $100 \mathrm{~m}$ and an aphotic zone below. Depending on the insolation based on the astronomical formula of Paltridge and Platt (1976), the light limitation function $I: \Omega \times[0,1] \rightarrow \mathbb{R}_{\geq 0}$ models the available light taking the ice cover and the exponential attenuation of water into account. Since the main part of the biological production occurs in the euphotic layer, particulate matter sinks from the euphotic layer to depth and remineralizes there according to the empirical power-law relationship (Martin et al., 1987).

The $\mathrm{N}$ model is the simplest biogeochemical model of the hierarchy and represents only phosphate $\left(\mathrm{PO}_{4}\right)$ as inorganic nutrients (cf. Bacastow and Maier-Reimer, 1990; Kriest et al., 2010). The available nutrients and light restrict the phytoplankton production (or biological uptake). The phytoplankton production

$f_{P}: \Omega \times[0,1] \rightarrow \mathbb{R}, f_{P}(x, t)=\mu_{P} y_{P}^{*} \frac{I(x, t)}{K_{I}+I(x, t)} \frac{\boldsymbol{y}_{N}(x, t)}{K_{N}+\boldsymbol{y}_{N}(x, t)}$ 
https://doi.org/10.5194/gmd-2021-392

Preprint. Discussion started: 7 January 2022

(c) Author(s) 2022. CC BY 4.0 License.

(c) (1)

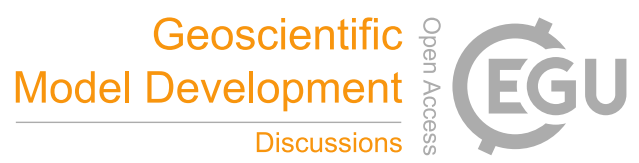

Table 2. Reference parameter values of the biogeochemical models taken from Kriest et al. (2010) as well as lower $\left(\boldsymbol{b}_{\ell}\right)$ and upper $\left(\boldsymbol{b}_{u}\right)$ bounds for the parameter values used to generate the Latin hypercube sample.

\begin{tabular}{|c|c|c|c|c|c|c|c|}
\hline Parameter & $\mathrm{N}$ & N-DOP & NP-DOP & NPZ-DOP & NPZD-DOP & $\boldsymbol{b}_{\ell}$ & $\boldsymbol{b}_{u}$ \\
\hline$k_{w}$ & 0.02 & 0.02 & 0.02 & 0.02 & 0.02 & 0.01 & 0.05 \\
\hline$k_{c}$ & & & 0.48 & 0.48 & 0.48 & 0.24 & 0.72 \\
\hline$\mu_{P}$ & 2.0 & 2.0 & 2.0 & 2.0 & 2.0 & 1.0 & 4.0 \\
\hline$\mu_{Z}$ & & & 2.0 & 2.0 & 2.0 & 1.0 & 4.0 \\
\hline$K_{N}$ & 0.5 & 0.5 & 0.5 & 0.5 & 0.5 & 0.25 & 1.0 \\
\hline$K_{P}$ & & & 0.088 & 0.088 & 0.088 & 0.044 & 0.176 \\
\hline$K_{I}$ & 30.0 & 30.0 & 30.0 & 30.0 & 30.0 & 15.0 & 60.0 \\
\hline$\sigma_{Z}$ & & & & 0.75 & 0.75 & 0.05 & 0.95 \\
\hline$\sigma_{\mathrm{DOP}}$ & & 0.67 & 0.67 & 0.67 & 0.67 & 0.05 & 0.95 \\
\hline$\lambda_{P}$ & & & 0.04 & 0.04 & 0.04 & 0.02 & 0.08 \\
\hline$\kappa_{P}$ & & & 4.0 & & & 2.0 & 6.0 \\
\hline$\lambda_{Z}$ & & & & 0.03 & 0.03 & 0.015 & 0.045 \\
\hline$\kappa_{Z}$ & & & & 3.2 & 3.2 & 1.6 & 4.8 \\
\hline$\lambda_{P}^{\prime}$ & & & 0.01 & 0.01 & 0.01 & 0.005 & 0.015 \\
\hline$\lambda_{Z}^{\prime}$ & & & & 0.01 & 0.01 & 0.005 & 0.015 \\
\hline$\lambda_{D}^{\prime}$ & & & & & 0.05 & 0.025 & 0.1 \\
\hline$\lambda_{\mathrm{DOP}}^{\prime}$ & & 0.5 & 0.5 & 0.5 & 0.5 & 0.25 & 1.0 \\
\hline$b$ & 0.858 & 0.858 & 0.858 & 0.858 & & 0.7 & 1.5 \\
\hline$a_{D}$ & & & & & 0.058 & 0.029 & 0.087 \\
\hline$b_{D}$ & & & & & 0.0 & 0.0 & 0.0 \\
\hline
\end{tabular}

depends on the maximum production rate $\mu_{P}$ and applies an implicitly prescribed concentration of phytoplankton $y_{P}^{*}=$ $0.0028 \mathrm{mmol} \mathrm{P} \mathrm{m}^{-3}$. Altogether, $n_{u}=5$ model parameters listed in Table 2 control the biogeochemical processes of the nutrient tracer $\boldsymbol{y}=\left(\boldsymbol{y}_{\mathrm{N}}\right)$.

The N-DOP model includes dissolved organic phosphorus (DOP) in addition to nutrients (N), i.e., $\boldsymbol{y}=\left(\boldsymbol{y}_{\mathrm{N}}, \boldsymbol{y}_{\mathrm{DOP}}\right)$ (cf. Bacastow and Maier-Reimer, 1991; Parekh et al., 2005; Kriest et al., 2010). This model computes the phytoplankton production also with (7) and introduces $n_{u}=7$ model parameters (Table 2).

The NP-DOP model contains phytoplankton $(\mathrm{P})$ in addition to nutrients $(\mathrm{N})$ and dissolved organic phosphorus (DOP), i.e., $\boldsymbol{y}=\left(\boldsymbol{y}_{\mathrm{N}}, \boldsymbol{y}_{\mathrm{P}}, \boldsymbol{y}_{\mathrm{DOP}}\right)$ (cf. Kriest et al., 2010). As a result of the explicit treatment of phytoplankton, the NP-DOP model computes the phytoplankton production again with (7) but using the explicit phytoplankton concentration $\boldsymbol{y}_{\mathrm{P}}$ instead of $y_{P}^{*}$. A quadratic 
loss term for phytoplankton, moreover, models the zooplankton grazing

$$
f_{Z}: \Omega \times[0,1] \rightarrow \mathbb{R}, f_{Z}(x, t)=\mu_{Z} y_{Z}^{*} \frac{\boldsymbol{y}_{P}(x, t)^{2}}{K_{P}^{2}+\boldsymbol{y}_{P}(x, t)^{2}}
$$

with the implicitly prescribed zooplankton concentration $y_{Z}^{*}=0.01 \mathrm{mmol} \mathrm{P} \mathrm{m}^{-3}$. The $n_{u}=13$ model parameters listed in Table 2 control the biogeochemical processes of the NP-DOP model.

The NPZ-DOP model consists of four tracers, nutrients $(\mathrm{N})$, phytoplankton $(\mathrm{P})$, zooplankton $(\mathrm{Z})$ and dissolved organic phosphorus (DOP), i.e., $\boldsymbol{y}=\left(\boldsymbol{y}_{\mathrm{N}}, \boldsymbol{y}_{\mathrm{P}}, \boldsymbol{y}_{\mathrm{Z}}, \boldsymbol{y}_{\mathrm{DOP}}\right)$ (cf. Kriest et al., 2010). While the phytoplankton production (7) is the same as for the NP-DOP model, the zooplankton grazing (8) explicitly contains the zooplankton concentration $\boldsymbol{y}_{Z}$ instead of the implicitly prescribed concentration $y_{Z}^{*}$. The model introduces $n_{u}=16$ model parameters summarized in Table 2 .

The NPZD-DOP model, the most complex model of the hierarchy, finally, contains detritus (D) in addition to nutrients (N), phytoplankton (P), zooplankton (Z) and dissolved organic phosphorus (DOP), i.e., $\boldsymbol{y}=\left(\boldsymbol{y}_{\mathrm{N}}, \boldsymbol{y}_{\mathrm{P}}, \boldsymbol{y}_{\mathrm{Z}}, \boldsymbol{y}_{\mathrm{D}}, \boldsymbol{y}_{\mathrm{DOP}}\right)$ (cf. Schmittner et al., 2005; Kriest et al., 2010). Both the phytoplankton production (7) and the zooplankton grazing (8) are identical to the NPZ-DOP model. Table 2 lists the $n_{u}=18$ model parameters of the NPZD-DOP model.

The MITgcm-PO4-DOP model contains phosphate $\left(\mathrm{PO}_{4}\right)$ and dissolved organic phosphorus (DOP). This model introduced by Dutkiewicz et al. (2005) for the biogeochemistry tutorial that uses the MIT general circulation model (cf. Marshall et al., 1997) resembles the N-DOP model. As the N-DOP model, the MITgcm-PO4-DOP introduces $n_{u}=7$ model parameters which we identified with the models parameters of the N-DOP model (Table 2).

\subsection{Transport matrix method}

The transport matrix method (TMM) reduces the simulation of the tracer transport of an offline model to matrix-vector multiplications. Khatiwala et al. (2005) applied a linear matrix equation instead of directly implementing a discretization scheme for the advection-diffusion equation (1) because the application of the operators A and D for the advection and diffusion on a spatially discretized tracer vector is linear (see also Khatiwala, 2007). Consequently, the TMM approximates the ocean circulation by matrices which include the influence of all parameterized processes represented in the underlying ocean circulation model on the transport.

Each time step of the simulation with the TMM requires only two matrix-vector multiplications and an evaluation of the biogeochemical model. For the discretization of the advection-diffusion equation, let $\left(x_{k}\right)_{k=1}^{n_{x}}$ a spatial discretization with $n_{x} \in \mathbb{N}$ grid points of the domain $\Omega$ (i.e., the ocean) and the time steps $t_{0}, \ldots, t_{n_{t}} \in[0,1], n_{t} \in \mathbb{N}$, specified by

$t_{j}:=j \Delta t, \quad j=0, \ldots, n_{t}, \quad \Delta t:=\frac{1}{n_{t}}$,

165 an equidistant grid of the time interval [0,1] (i.e., one model year). For a time instant $t_{j}, j \in\left\{0, \ldots, n_{t}-1\right\}$, the vector $\boldsymbol{y}_{j i} \approx$ $\left(y_{i}\left(t_{j}, x_{k}\right)\right)_{k=1}^{n_{x}} \in \mathbb{R}^{n_{x}}$ is a spatial discretization of the tracer $y_{i}, i \in\left\{1, \ldots, n_{y}\right\}$, and $\boldsymbol{q}_{j i} \approx\left(q_{i}\left(x_{k}, t_{j}, \boldsymbol{y}_{j}, \boldsymbol{u}\right)\right)_{k=1}^{n_{x}} \in \mathbb{R}^{n_{x}}$ the spatially discretized biogeochemical term $q_{i}$ for the tracer $y_{i}$. Besides, $\boldsymbol{y}_{j}:=\left(\boldsymbol{y}_{j i}\right)_{i=1}^{n_{y}} \in \mathbb{R}^{n_{y} n_{x}}$ and $\boldsymbol{q}_{j}:=\left(\boldsymbol{q}_{j i}\right)_{i=1}^{n_{y}} \in \mathbb{R}^{n_{y} n_{x}}$ summarize the tracer discretization as well as the biogeochemical terms for all tracers using a reasonable concatenation. 
Discretizing the advection and horizontal diffusion explicitly and the vertical diffusion implicitly, the application of a semidiscrete Euler scheme for (1) yields a time-stepping

$\boldsymbol{y}_{j+1}=\left(\mathbf{I}+\Delta t \mathbf{A}_{j}+\Delta t \mathbf{D}_{j}^{h}\right) \boldsymbol{y}_{j}+\Delta t \mathbf{D}_{j}^{v} \boldsymbol{y}_{j+1}+\Delta t \boldsymbol{q}_{j}\left(\boldsymbol{y}_{j}, \boldsymbol{u}\right), \quad j=0, \ldots, n_{t}-1$,

with the identity matrix $\mathbf{I} \in \mathbb{R}^{n_{x} \times n_{x}}$ and the spatially discretized counterparts $\mathbf{A}_{j}, \mathbf{D}_{j}^{h}$ and $\mathbf{D}_{j}^{v}$ of the operators $A, D_{h}$ and $D_{v}$ at time instant $t_{j}, j \in\left\{0, \ldots, n_{t}-1\right\}$. The matrices $\mathbf{D}_{j}^{v}$ are block-diagonal since they involve only the vertical part of the diffusion. Thus, each water column is separated from the others. We denote the explicit and implicit transport matrices by

$\mathbf{T}_{j}^{\exp }:=\mathbf{I}+\Delta t \mathbf{A}_{j}+\Delta t \mathbf{D}_{j}^{h} \in \mathbb{R}^{n_{x} \times n_{x}}$

$\mathbf{T}_{j}^{\mathrm{imp}}:=\left(\mathbf{I}-\Delta t \mathbf{D}_{j}^{v}\right)^{-1} \in \mathbb{R}^{n_{x} \times n_{x}}$

for each time instant $t_{j}, j \in\left\{0, \ldots, n_{t}-1\right\}$. The implicit matrices $\mathbf{T}_{j}^{\mathrm{imp}}, j \in\left\{0, \ldots, n_{t}-1\right\}$, are block-diagonal again since the inversion of a matrix keeps this structure. Finally, a time step of the marine ecosystem model using the TMM is given by

$\boldsymbol{y}_{j+1}=\mathbf{T}_{j}^{\mathrm{imp}}\left(\mathbf{T}_{j}^{\mathrm{exp}} \boldsymbol{y}_{j}+\Delta t \boldsymbol{q}_{j}\left(\boldsymbol{y}_{j}, \boldsymbol{u}\right)\right)=: \varphi_{j}\left(\boldsymbol{y}_{j}, \boldsymbol{u}\right), \quad j=0, \ldots, n_{t}-1$

Due to the grid-point based ocean circulation model, both the explicit and the implicit transport matrices are sparse. For the implicit ones, this is given by their block-diagonal structure.

In practical computations, the TMM computed and stored monthly averaged matrices and interpolated those linearly for any time instant $t_{j}, j=0, \ldots, n_{t}-1$. In this paper, we applied transport matrices computed with the MIT ocean model (Marshall et al., 1997) used a global configuration with a latitudinal and longitudinal resolution of $2.8125^{\circ}$ and 15 vertical layers.

\subsection{Computation of steady annual cycles}

For a marine ecosystem model, the steady annual cycle is a fixed-point of the spin-up. Applying the above iteration (9) over one model year, the steady annual cycle (i.e., an annual periodic solution) in a fully discrete setting fulfills

$\boldsymbol{y}_{n_{t}}=\boldsymbol{y}_{0}$.

The steady annual cycle is a fixed-point of the nonlinear mapping

$\Phi:=\varphi_{n_{t}-1} \circ \ldots \circ \varphi_{0}$

with $\varphi_{j}$ defined in (9), describing the time integration of (9) over one model year. A classical fixed-point iteration takes the form

$\boldsymbol{y}^{\ell+1}=\Phi\left(\boldsymbol{y}^{\ell}, \boldsymbol{u}\right), \quad \ell=0,1, \ldots$

using an arbitrary start vector $\boldsymbol{y}^{0} \in \mathbb{R}^{n_{y} n_{x}}$ and model parameters $\boldsymbol{u} \in \mathbb{R}^{n_{u}}$. Interpreting the fixed-point iteration as pseudo-time stepping or spin-up, the vector $\boldsymbol{y}^{\ell} \in \mathbb{R}^{n_{y} n_{x}}$ contains the tracer concentrations at the first time instant of the model year $\ell \in \mathbb{N}$. 
We tested the numerical convergence of the spin-up (i.e,. of the iteration (10)) with the difference between two consecutive iterates determined by

$\varepsilon_{\ell}:=\left\|\boldsymbol{y}^{\ell}-\boldsymbol{y}^{\ell-1}\right\|$

for iteration (model year) $\ell \in \mathbb{N}_{0}$. For this purpose, we quantified this difference with various norms. Namely, we defined a weighted Euclidean norm

$\|\boldsymbol{z}\|_{2, w}:=\left(\sum_{i=1}^{n_{y}} \sum_{k=1}^{n_{x}} w_{k} z_{i k}^{2}\right)^{\frac{1}{2}}$

with weights $w_{k} \in \mathbb{R}_{>0}$ for $k \in\left\{1, \ldots, n_{x}\right\}$ and $\boldsymbol{z} \in \mathbb{R}^{n_{y} n_{x}}$ indexed as $\boldsymbol{z}=\left(\left(z_{i k}\right)_{k=1}^{n_{x}}\right)_{i=1}^{n_{y}}$. If all weights are equal to 1 (i.e., $w_{k}=1$ for $\left.k=1, \ldots, n_{x}\right)$, the norm $\|\cdot\|_{2, w}$ corresponds to the Euclidean norm $\|\cdot\|_{2}$. We denoted by $\|\cdot\|_{2, V}$ the discretized counterpart of the $L^{2}(\Omega)^{n_{y}}$ norm using the weights $w_{k}=\left|V_{k}\right|, k=1, \ldots, n_{x}$, with the box volume $\left|V_{k}\right|$ corresponding to the grid point $x_{k}$. In order to consider not only the difference at first time instant but for the whole trajectory over one model year, we, moreover, defined the weighted Euclidean norm $\|\cdot\|_{2, w, T}$ by

$\|\boldsymbol{z}\|_{2, w, T}:=\left(\sum_{i=1}^{n_{y}} \sum_{j=0}^{n_{t}-1} \Delta t \sum_{k=1}^{n_{x}} w_{k} z_{j i k}^{2}\right)^{\frac{1}{2}}$

for a weight vector $\boldsymbol{w} \in \mathbb{R}_{>0}^{n_{x}}$ and $\boldsymbol{z} \in \mathbb{R}^{n_{t} n_{y} n_{x}}$ indexed as $\boldsymbol{z}=\left(\left(\left(z_{j i k}\right)_{k=1}^{n_{x}}\right)_{i=1}^{n_{y}}\right)_{j=0}^{n_{t}-1}$. Analogous to the weighted Euclidean norm $\|\cdot\|_{2, w}$, we denoted by $\|\cdot\|_{2, T}$ the Euclidean norm and by $\|\cdot\|_{2, V, T}$ the weighted Euclidean norm $\|\cdot\|_{2, w, T}$ using weights $w_{k}=\left|V_{k}\right|$ for $k \in\left\{1, \ldots, n_{x}\right\}$. In addition to the norms including all grid points of the discretization $\left(x_{k}\right)_{k=1}^{n_{x}}$, we restricted norms to horizontal layers of the ocean discretization, such as to the upper layer describing the ocean surface. We identified with $\left.\|\cdot\|\right|_{L}$ for $L \subset\{1, \ldots, 15\}$ the restriction of norm $\|\cdot\|$ to the layers selected in the set $L$. For example, $\left.\|\cdot\|\right|_{L}$ with $L:=\{1\}$ restricted the norm to the grid points of the ocean surface.

\subsection{Temporal coarsening of transport matrices}

215 Using simple matrix operations, transport matrices computed with a given time step can be used to generate matrices corresponding to a bigger time step. The procedure has been described in Khatiwala (2007). Following his approach, we used the transport matrices $\mathbf{T}_{j}^{\mathrm{exp}}$ and $\mathbf{T}_{j}^{\mathrm{imp}}, j \in\left\{0, \ldots, n_{t}-1\right\}$, to generate transport matrices

$\mathbf{T}_{j, m}^{\exp }:=\mathbf{I}+m\left(\mathbf{T}_{j}^{\exp }-\mathbf{I}\right)$,

$\mathbf{T}_{j, m}^{\mathrm{imp}}:=\left(\mathbf{T}_{j}^{\mathrm{imp}}\right)^{m}$

corresponding to a time step with coarsening factor $m \in \mathbb{N}$. Consequently, these matrices represent larger time steps than the ones in the underlying ocean circulation model which were used to generate the transport matrices $\mathbf{T}_{j}^{\mathrm{exp}}$ and $\mathbf{T}_{j}^{\mathrm{imp}}$. The explicit transport matrix $\mathbf{T}_{j, m}^{\exp }$ is the exact representation of the larger time step, i.e.,

$\mathbf{T}_{j, m}^{\exp }=\mathbf{I}+m\left(\mathbf{T}_{j}^{\exp }-\mathbf{I}\right)=\mathbf{I}+m\left(\mathbf{I}+\Delta t \mathbf{A}_{j}+\Delta t \mathbf{D}_{j}^{h}-\mathbf{I}\right)=\mathbf{I}+m \Delta t \mathbf{A}_{j}+m \Delta t \mathbf{D}_{j}^{h}$. 
The implicit transport matrix $\mathbf{T}_{j, m}^{\mathrm{imp}}$ is an approximation with a loss of accuracy of order $\Delta t^{2}$ since (using the binomial theorem)

$$
\begin{aligned}
\mathbf{T}_{j, m}^{\mathrm{imp}} & =\left(\mathbf{T}_{j}^{\mathrm{imp}}\right)^{m}=\left(\left(\mathbf{I}-\Delta t \mathbf{D}_{j}^{v}\right)^{-1}\right)^{m}=\left(\left(\mathbf{I}-\Delta t \mathbf{D}_{j}^{v}\right)^{m}\right)^{-1}=\left(\sum_{k=0}^{m}\left(\begin{array}{c}
m \\
k
\end{array}\right) \mathbf{I}^{m-k}\left(-\Delta t \mathbf{D}_{j}^{v}\right)^{k}\right)^{-1} \\
& =\left(\left(\begin{array}{c}
m \\
0
\end{array}\right) \mathbf{I}^{m}\left(-\Delta t \mathbf{D}_{j}^{v}\right)^{0}+\left(\begin{array}{c}
m \\
1
\end{array}\right) \mathbf{I}^{m-1}\left(-\Delta t \mathbf{D}_{j}^{v}\right)^{1}+\sum_{k=2}^{m}\left(\begin{array}{c}
m \\
k
\end{array}\right) \mathbf{I}^{m-k}\left(-\Delta t \mathbf{D}_{j}^{v}\right)^{k}\right)^{-1} \\
& =\left(\mathbf{I}-m \Delta t \mathbf{D}_{j}^{v}+\sum_{k=2}^{m}\left(\begin{array}{c}
m \\
k
\end{array}\right) \mathbf{I}^{m-k}\left(-\Delta t \mathbf{D}_{j}^{v}\right)^{k}\right)^{-1}=\left(\left(\mathbf{I}-m \Delta t \mathbf{D}_{j}^{v}\right)+\mathcal{O}(\Delta t)^{2}\right)^{-1}
\end{aligned}
$$

(cf. Piwonski and Slawig, 2016b; Khatiwala, 2007). These transport matrices are still sparse (Khatiwala, 2007).

The time step of the ocean circulation model used for the computation of the transport matrices $\mathbf{T}_{j}^{\text {exp }}$ and $\mathbf{T}_{j}^{\text {imp }}, j \in$ per model year is $n_{t}=2880$. We, hereinafter, denoted this time step with $1 \Delta t$ using $\Delta t=\frac{1}{2880}$. More specifically, time step $2 \Delta t$ corresponds to the coarsening factor 2, i.e., a doubling of the effective time step, with $n_{t}=1440$. In order to identify the time step used to run the spin-up (10), we denoted by

$\boldsymbol{y}^{\ell+1, m}=\Phi^{m}\left(\boldsymbol{y}^{\ell}, \boldsymbol{u}\right), \quad \ell=0,1, \ldots$,

235 the time-integration of one model year using the time step $m \Delta t$ with the factor $m \in \mathbb{N}$ for $\boldsymbol{y}^{\ell} \in \mathbb{R}^{n_{y} n_{x}}$ and $\boldsymbol{u} \in \mathbb{R}^{n_{u}}$. More importantly, the transport matrices $\mathbf{T}_{j, m}^{\exp }$ and $\mathbf{T}_{j, m}^{\mathrm{imp}}, j \in\left\{0, \ldots, n_{t}-1\right\}$, enter (9) for the application of the time step $m \Delta t$ instead of the transport matrices $\mathbf{T}_{j}^{\mathrm{exp}}$ and $\mathbf{T}_{j}^{\mathrm{imp}}$.

Any choice $m \in \mathbb{N}$ is possible in the above computations. For every value of $m$ to be used, twelve pairs of explicit and implicit transport matrices, however, have to be supplied. Since we wanted to check a rather wide variability of the time steps, we have chosen the possible coarsening factors

$m \in M:=\{1,2,4,8,16,32,64\}$

in this study. The upper limit of $m=64$ was motivated by our observation that the spin-up for most of the models did not converge anymore for larger time steps.

\subsection{Negative tracer concentrations}

245 A time step that is too large can cause negative tracer concentrations. Given a non-negative tracer distribution $\boldsymbol{y}_{j}$ in all grid points at a time instant $t_{j}, j \in\left\{0, \ldots, n_{t}-1\right\}$, the multiplication with the explicit transport matrix $\mathbf{T}_{j}^{\exp }$ in (9) will always result in a non-negative distribution. In contrast, the source term $\boldsymbol{q}_{j}\left(\boldsymbol{y}_{j}, \boldsymbol{u}\right)$ may give negative values for some tracers at some points even though the input variable $\boldsymbol{y}_{j}$ is non-negative. In many models, the latter is enforced by a simple setting of negative values to zero before the evaluation of the source term. Such a setting obviously increases the total mass in the ecosystem. Hence, a frequent occurrence of big negative values and their correction to zero will result in a changed steady solution obtained in the spin-up. Despite of such a correction before the source term step, the sum in the bracket in (9) might be negative at some 
https://doi.org/10.5194/gmd-2021-392

Preprint. Discussion started: 7 January 2022

(c) Author(s) 2022. CC BY 4.0 License.

(c) (i)

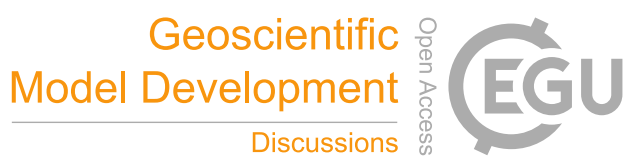

points, depending on the used time step. Thus, after applying the implicit matrix, the result of the time step $\boldsymbol{y}_{j+1}$ might contain negative values as well.

Small negative values in some points do not necessarily change the convergence of a spin-up to a steady annual cycle.

Therefore, a main criterion for algorithms that use bigger time steps (as the ones presented here) is if the spin-up converges, and if it converges to the same solution as for the standard setting. We also investigated an algorithm that enforces non-negative tracer concentrations and how this effects the solution and the cost saving.

\section{Step size control algorithms}

Methods for automatic step size control automatically adapt the time step during the calculation of a transient computation. They may, therefore, also be used for the steady annual cycle computation via a spin-up. A step size control method estimates the local discretization error by computing two approximations, either with different time steps (in the method used here) or by using two different time integration methods. The approach used here is based on the Richardson extrapolation (Richardson and Glazebrook, 1911; Richardson and Gaunt, 1927). For the estimate of the local discretization error, the step size control computes two approximations with two different time step sizes in every step. Depending on the error estimate and a desired accuracy, the step size control accepts or rejects the approximation calculated with the smaller time step (the approximation computed with the larger time step always serves the error estimation only). Then, it adapts the step size to a value that, using the estimation of the error, would result in the desired accuracy. Thus, in this step, an increase or a decrease of the step size is possible. Using the adapted step size, the method, afterwards, either starts the calculation of the next step or reruns the calculation of the current step. In summary, the step size control finally uses always the largest time step that keeps the error below the given tolerance.

Step size control methods are based on the existence of an asymptotic expansion of the discretization error (Stoer and Bulirsch, 2002, Sect. 7.2.3). To obtain this result, the unknown solution is assumed to be three times continuously differentiable in time. The question whether this regularity is satisfied for the models investigated in this work is not discussed here. We just note that all models have continuous right-hand sides (i.e., source-minus-sink terms). Furthermore, it is quite usual to apply a step size control even though some assumptions of the underlying mathematical theory may not be given or shown for an actual application. Since we compared our results obtained with the step size control to those with constant time steps, an assessment of the method is possible.

Algorithm 1 depicts the step size control for the calculation of a steady annual cycle. It is based on Deuflhard and Bornemann (2013, Algorithm 5.2). Since we are aiming at a steady annual cycle obtained in a spin-up, we do not check for the local discretization error after every single time step, but only after an adjustable number of model years. This number $\left(n_{s} \in \mathbb{N}\right)$ is one of the input parameters of the algorithm. The loop over the $n_{s}$ years is realized in lines 10 to 13. Another important feature is the set of possible or desired multiplication factors $m$. Here, the $m=\max M$ value is inadmissible because the step size control estimates the local error using two approximations calculated with the selected time step $m \Delta t$ and a larger time step. Thus, the set $M \backslash \max M$ defines the admissible time steps of Algorithm 1. 


\section{Algorithm 1 Step size control.}

Input: Initial concentration: $\mathbf{y}^{0} \in \mathbb{R}^{n_{y} n_{x}}$,

Parameter vector: $\mathbf{u} \in \mathbb{R}^{n_{u}}$,

Number of model years: $T \in \mathbb{N}$,

Coarsening factor of the initial time step: $m_{\text {init }} \in M \backslash \max M$,

Number of model years for the error estimation: $n_{s} \in \mathbb{N}$,

Tolerance: $\tau_{0} \in \mathbb{R}_{>0}$

Output: $\mathbf{y}^{T} \in \mathbb{R}^{n_{y} n_{x}}$ (superscript referring to $\ell=T$ here, not meaning transposed vector)

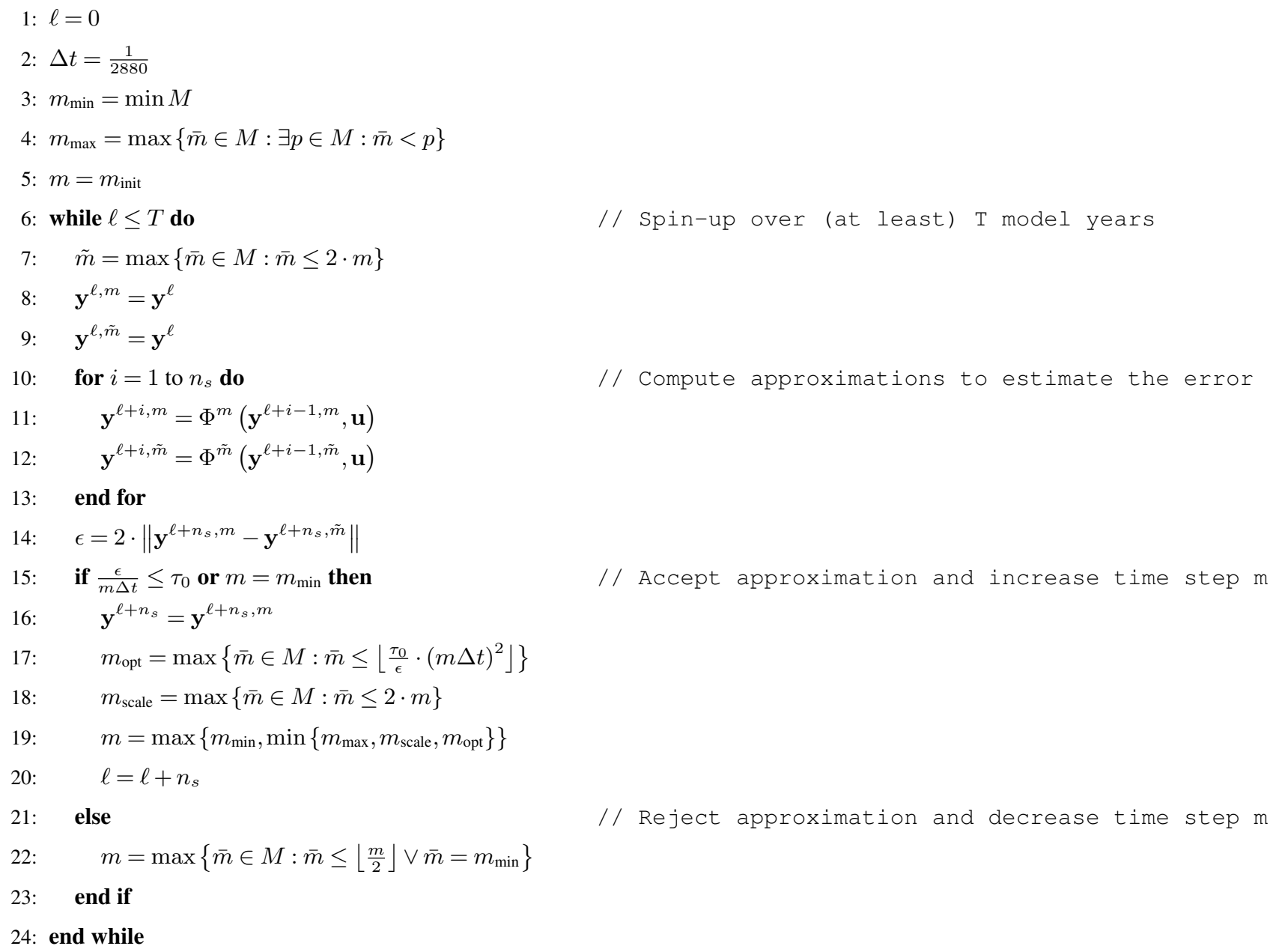

We designed the step size control algorithm having various options. The user may select, firstly, the initial time step $m_{\text {init }} \Delta t$ of the step size control with $m_{\text {init }} \in M \backslash \max M$. The second option is the number $n_{s} \in \mathbb{N}$ of model years after which the error is estimated. The step size control computes the two approximations with time steps $m \Delta t$ and $\tilde{m} \Delta t$ for the model year $\ell+n_{s}, \ell \in \mathbb{N}_{0}$, and $m, \tilde{m} \in M$ with $m<\tilde{m}$ and estimates the local discretization error $\left\|\mathbf{y}^{\ell+n_{s}, m}-\mathbf{y}^{\ell+n_{s}, \tilde{m}}\right\|$. The choice of 


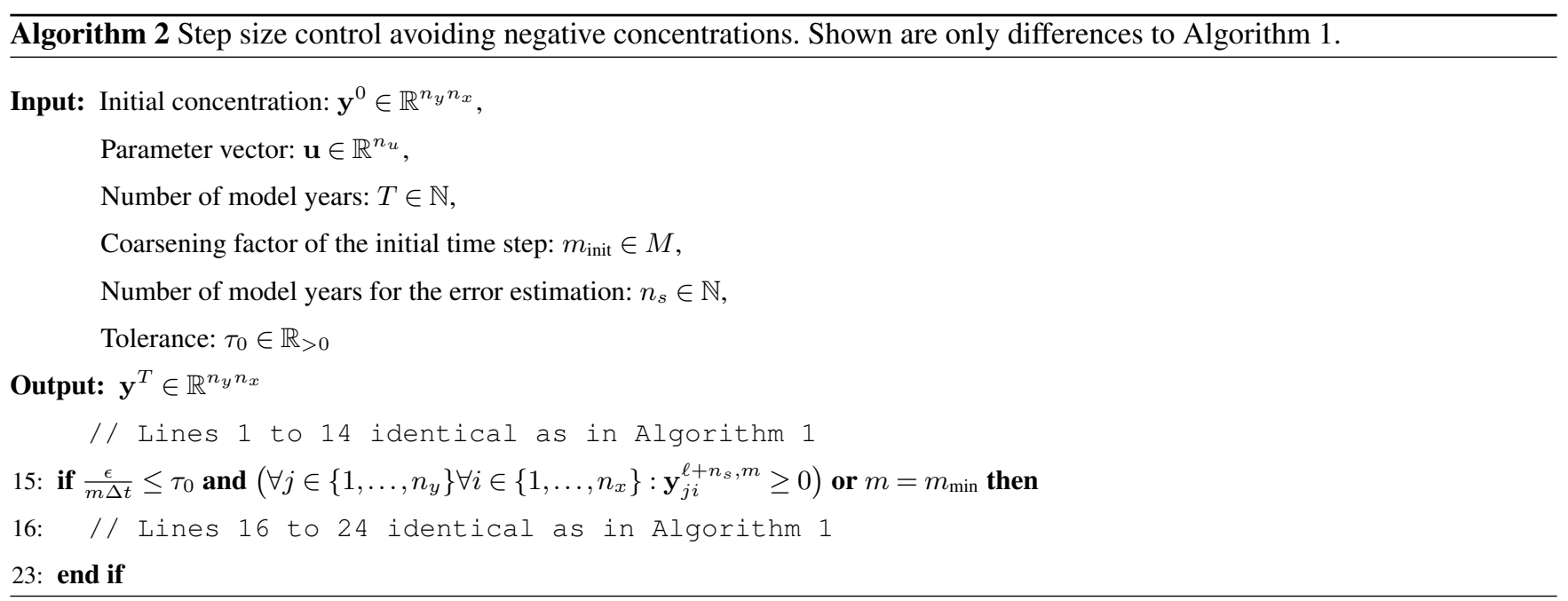

the next time step depends on this error (line 15 or 21 and following). Thirdly, it is possible to choose the norm that may have an influence on the error estimation. Another setting of the step size control, fourthly, is the tolerance $\tau_{0} \in \mathbb{R}_{>0}$ which controls the acceptance of the approximation. By default, the step size control applies the coarsening factor $m_{\text {init }}=1$ for the initial time step $m_{\text {init }} \Delta t, n_{s}=1$, the volume-weighted Euclidean norm $\|\cdot\|_{2, V}$ and the tolerance $\tau_{0}=1$.

A variant of Algorithm 1 includes an additional avoidance of negative concentrations (see Algorithm 2). If enabled, the step size control accepts an approximation - as additional criterion to the local discretization error - if and only if the concentration of this approximation is non-negative at all grid points $x_{k}, k \in\left\{1, \ldots, n_{x}\right\}$, or if the approximation was calculated with the smallest possible time step. Otherwise, the step size control reruns this step with a decreased time step.

\section{Decreasing time steps algorithm}

The step size control algorithms described above require an increased computational effort to automatically adjust the time step. They both try to find the optimal step size in the sense that it should be as big as possible but still keeps the local discretization error below the given tolerance. Therefore, the algorithms are automatically able to both increase and decrease the time step during the spin-up. This optimality comes with the additional effort of computing always two approximations to estimate this error.

The decreasing time steps algorithm presented in this section, in contrast, exclusively decreases the time step during the spin-up. The motivation is the same, namely to reduce the computational costs. The algorithm assumes that in its beginning bigger time steps are sufficient. The procedure is shown in Algorithm 3. Starting from an initial time step $m_{\text {init }} \Delta t$ with a coarsening factor $m_{\text {init }} \in M$, the algorithm uses the same time step until the norm $\left\|\mathbf{y}^{\ell}-\mathbf{y}^{\ell+n_{s}}\right\|, \ell \in \mathbb{N}_{0}$, fell below a given tolerance $\varepsilon \in \mathbb{R}_{>0}$. In other words, the spin-up calculation with this time step already reached almost a steady annual cycle. If the norm fell below the tolerance, the algorithm decreases the time step. The assessment whether a significant reduction was 


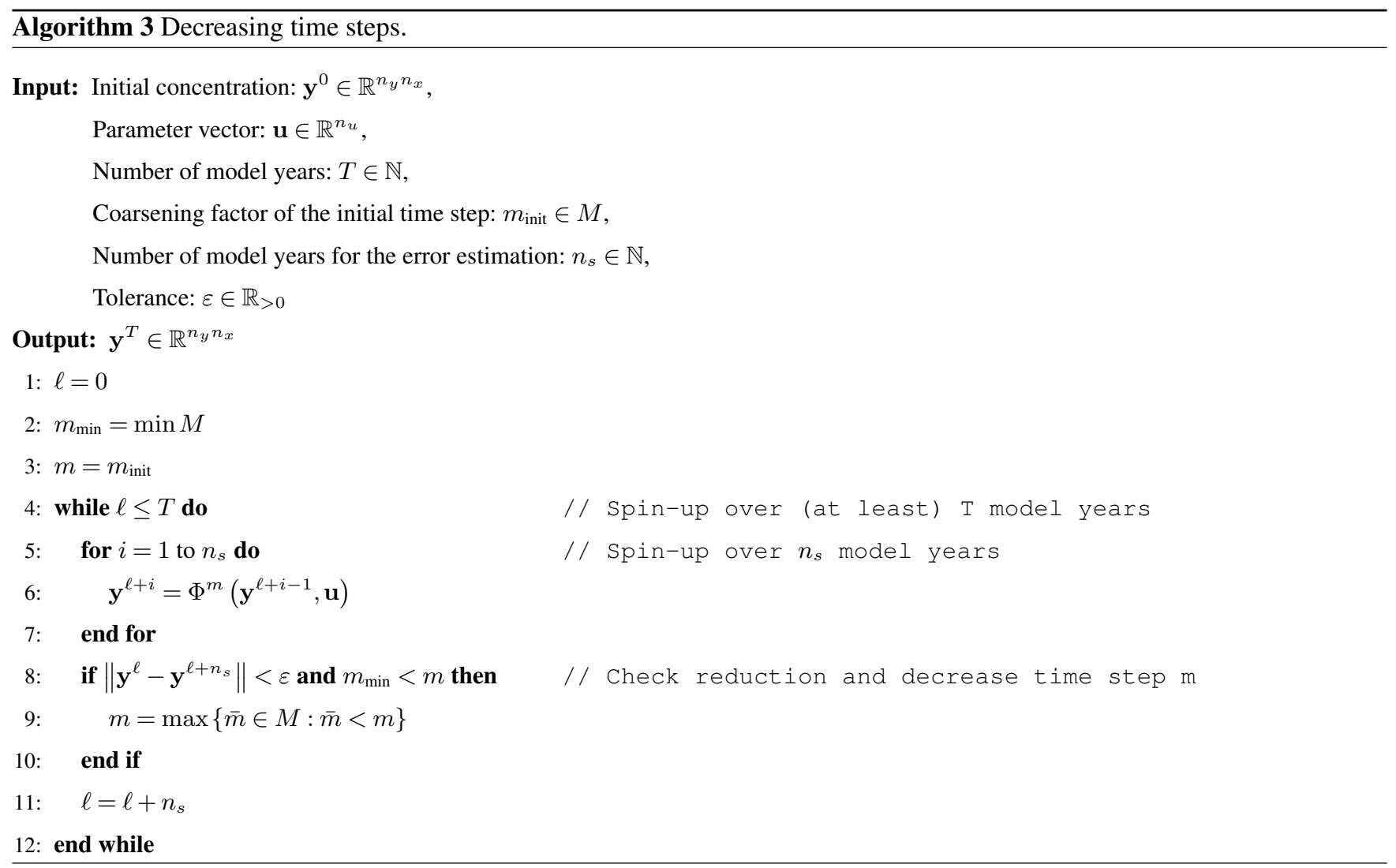

still achieved with the current time step takes place after a fixed number of $n_{s} \in \mathbb{N}$ model years. By default, the decreasing time steps algorithm uses the initial time step $m_{\text {init }} \Delta t$ with the coarsening factor $m_{\text {init }}=64, n_{s}=50$ and the tolerance $\varepsilon=0.001$.

\section{Results}

The automatic adjustment of the time step used for the spin-up computation influenced both the computational effort and the accuracy of the approximation of the steady annual cycle. In this section, we present the numerical results using the adaptive step size control (see Sect. 3) and the decreasing time steps algorithm (Sect. 4) to shorten the runtime of the computation of the steady annual cycle. We assessed the accuracy and cost saving of the calculated approximations.

\subsection{Experimental setup}

For each part of the spin-up calculation (i.e., $n_{s} \in \mathbb{N}$ model years), we used the marine ecosystem toolkit for optimization and simulation in 3D (Metos3D, Piwonski and Slawig, 2016a, b). Overall, we computed all spin-ups over 10000 model years. We started the spin-up always with a constant global mean tracer concentration of $2.17 \mathrm{mmol} \mathrm{P} \mathrm{m}^{-3}$ for $\mathrm{PO}_{4}$ and, if present, $0.0001 \mathrm{mmol} \mathrm{P} \mathrm{m}^{-3}$ for the tracers DOP, $\mathrm{P}, \mathrm{Z}$ and $\mathrm{D}$. 
Using different parameter vectors, we calculated the steady annual cycles for the various biogeochemical models. We used, on the one hand, the reference parameter vectors listed in Table 2 and, on the other hand, 100 parameter vectors generated by a Latin hypercube sample within the bounds defined in Table 2 for each biogeochemical model (cf. McKay et al., 1979). We created these parameter vectors by the lhs routine of Lee (2014).

For the assessment of the calculated approximations of the steady annual cycle, we compared them with reference solutions. These were chosen as the result obtained by a spin-up using Metos3D with constant time step $m \Delta t, m \in M$, also over 10000 model years. These solutions are denoted by $\mathbf{y}^{10000, m}$ and described in more detail in Pfeil and Slawig (2021a). In most cases, we considered the case $m=1$ only. The reference solutions were also used to measure, in particular, the accuracy of an approximation $\mathbf{x} \in \mathbb{R}^{n_{y} n_{x}}$ obtained by one of the algorithms (i.e., the step size control or the decreasing time steps algorithm) by the relative difference

$\frac{\left\|\mathbf{x}-\mathbf{y}^{10000,1}\right\|_{2}}{\left\|\mathbf{y}^{10000,1}\right\|_{2}}$

We call this quantity (13) the (relative) error of the respective result $\mathrm{x}$. Furthermore, we quantified the saving of the computational costs by

$\frac{c_{\text {ref }}-c_{\text {algo }}}{c_{\text {ref }}}$,

where $c_{\text {ref }} \in \mathbb{N}$ denotes the number of model evaluations used to compute the reference solution (i.e., $c_{\text {ref }}=10000 \cdot 2880$, according to the $n_{t}=2880$ time steps for each of the 10000 model years) and $c_{\text {algo }} \in \mathbb{N}$ denotes the number of model evaluations of the respective algorithm.

\subsection{Step size control algorithms}

In this section, we present the results using the step size control to compute a steady annual cycle (Algorithms 1 and 2). The step size control applies different time steps to adapt the step size using an estimation of the local discretization error. Starting with a default setting for Algorithm 1 (Sect. 5.2.1), we also analyzed the behavior using the avoidance of negative tracer concentrations using Algorithm 2 (Sect. 5.2.2) and, briefly, other configuration settings (Sect. 5.2.3) defined in Sect. 3.

\subsubsection{Algorithm 1 with default step size control setting}

For the reference parameter vectors of Table 2, the step size control algorithm with default setting computed approximations of the steady annual cycle that were almost identical to the ones obtained with a constant step size. For the N, N-DOP and MITgcm-PO4-DOP models, the step size control utilized the largest possible time step by increasing the time step in the first steps to $32 \Delta t$. Accordingly, the algorithm lowered the computational costs by $95 \%$. In contrast, the step size control used always time step $1 \Delta t$ (i.e., no increase of the time step occurred) for the other three biogeochemical models NP-DOP, NPZDOP and NPZD-DOP. Therefore, the computational effort for these three models was greater than for the solution $\mathbf{y}^{10000,1}$.

350 The value computed with formula (14) was -0.5. Obviously, the obtained solution was the same as the one using constantly $1 \Delta t$. As an example, Fig. 1 indicates the similar convergence behavior towards a steady annual cycle using the step size control 


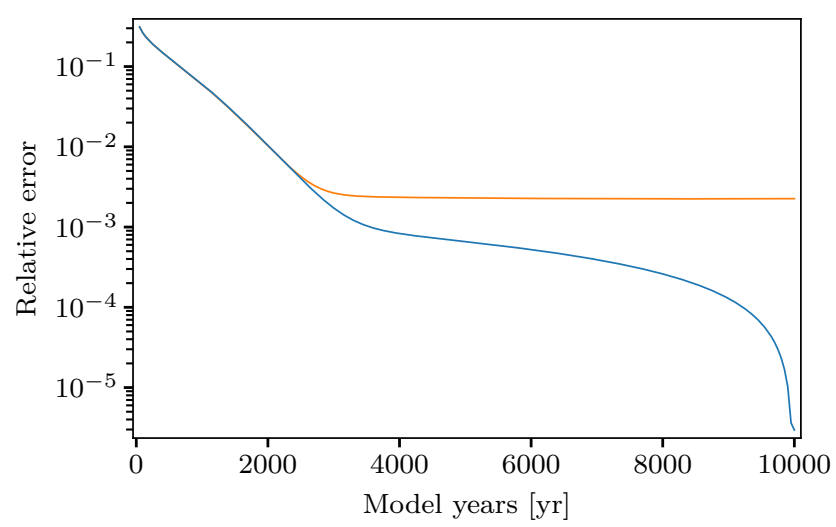

Figure 1. Results of spin-up computations for the reference parameter vector (see Table 2) and the N model using the step size control with default setting (Algorithm 1) and with avoidance of negative concentrations (Algorithm 2). Shown are the convergence of the spin-up (left), i.e., the norms of the difference (11) between consecutive iterations, and the relative error (13) (right). On the left, the curves for the reference run and the one with step size control avoiding negative concentrations overlap.

and the constant step size $1 \Delta t$, both for the $\mathrm{N}$ model. The convergence behavior using the step size control concurred with the one of the spin-up with constant time step $32 \Delta t$, i.e. $\mathbf{y}^{10000,32}$. In this case, the step size control doubled the time step five times up to the maximal time step $32 \Delta t$ in the first five model years. Afterwards, the time step remained unchanged for the entire spin-up (cf. Pfeil and Slawig, 2021a, Figs. 1 and 4). Consequently, the accuracy of this approximation resembled that of $\mathbf{y}^{10000,32}$ (Fig. 1, right). The step size control resulted in a cost saving of $95 \%$ compared to the solution $\mathbf{y}^{10000,1}$. Indeed, the cost saving was even $97 \%$ using directly the spin-up with time step $32 \Delta t$ because the step size control necessitated two approximations calculated with different time steps to estimate the local error for every model year. Looking at the reduction, it would be possible to terminate the step size control even after a much lower number of model years to lower further the computational effort (Fig. 1). For the N-DOP and MITgcm-PO4-DOP model, the results were similar.

Also for the 100 parameter vectors of the Latin hypercube sample, Algorithm 1 yielded solutions that showed the same accuracy as solutions obtained with a fixed step size. For the N, N-DOP and MITgcm-PO4-DOP models, the results were consistent with those for the reference parameter vectors above, i.e., the accuracy of the approximations coincided with that for the spin-up calculated with constant time step $32 \Delta t$ (Figs. 2 and 3). The algorithm increased the time step up to the maximum of $32 \Delta t$ and, afterwards, used only this time step. For a few parameter vectors, the step size control, however, did not increase the time step directly, but computed several model years before increasing the time step. For these three models, the step size control resulted in a tremendous cost saving (Fig. 4). For the NP-DOP, NPZ-DOP and NPZD-DOP models and more than 85 parameter vectors, the algorithm did not increase the time step. Thus, the approximations corresponded to the reference solution $\mathbf{y}^{10000,1}$ (Fig. 2) in these cases. For the remaining parameter vectors, at least one increase and, possibly, some decreases of the time step took place. This resulted in a larger relative error but still a reasonable accuracy of the calculated approximation. Except for a few parameter vectors where the step size control increased the time step, the step size control 

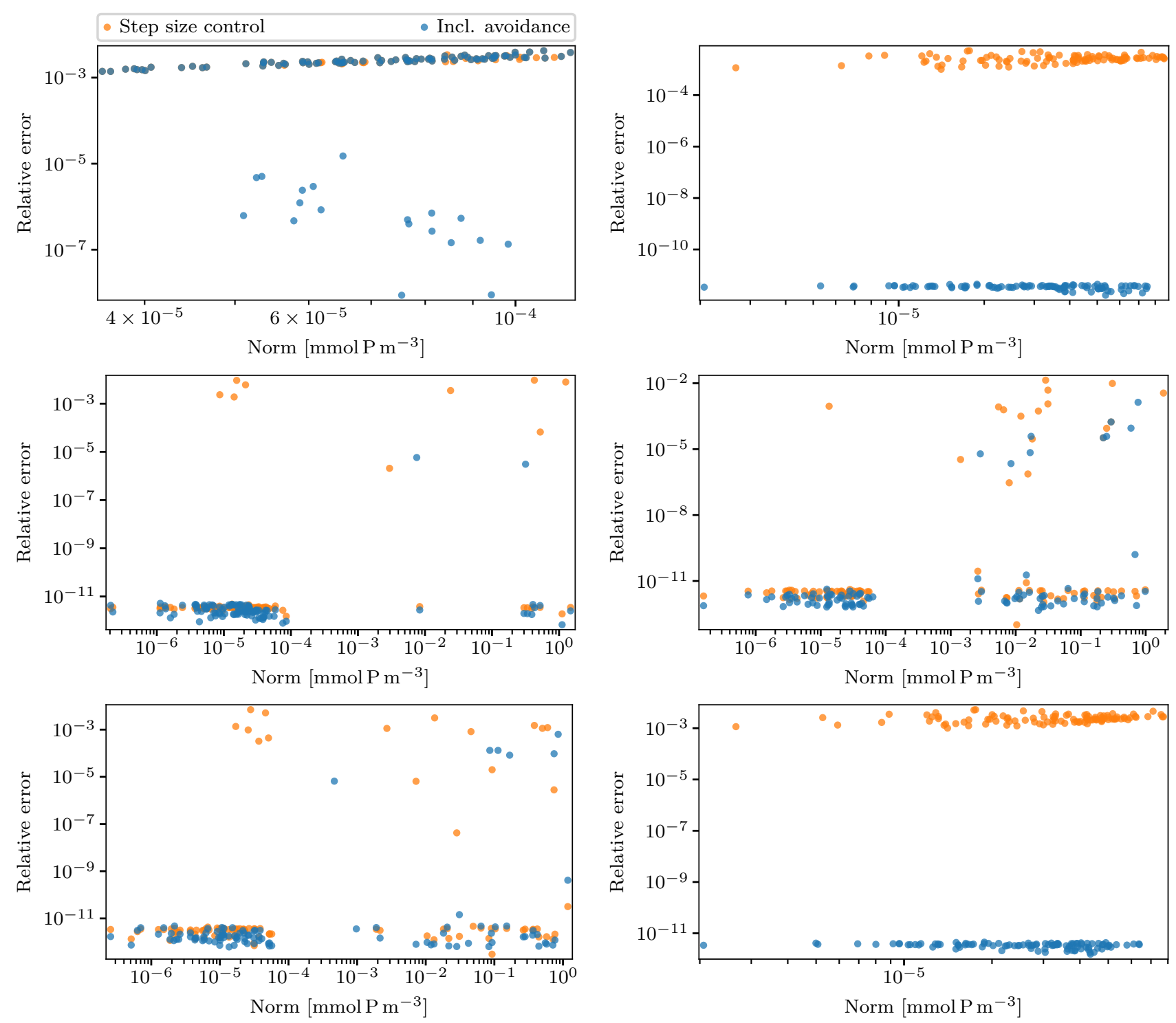

Figure 2. Norm of difference (11) and relative error (13) for $\ell=10000$ using the step size control with the default setting and with avoidance of negative concentrations. Shown are the results for the different biogeochemical models and all parameter vectors of the Latin hypercube sample (top left: N model, top right: N-DOP model, middle left: NP-DOP model, middle right: NPZ-DOP model, bottom left: NPZD-DOP model, bottom right: MITgcm-PO4-DOP model). Some points of the results using the avoidance of negative concentrations obscured the points of the step size control results.

caused a larger computational effort than using $1 \Delta t$ constantly because the algorithm needs two approximations to estimate the local discretization error (Fig. 4). 

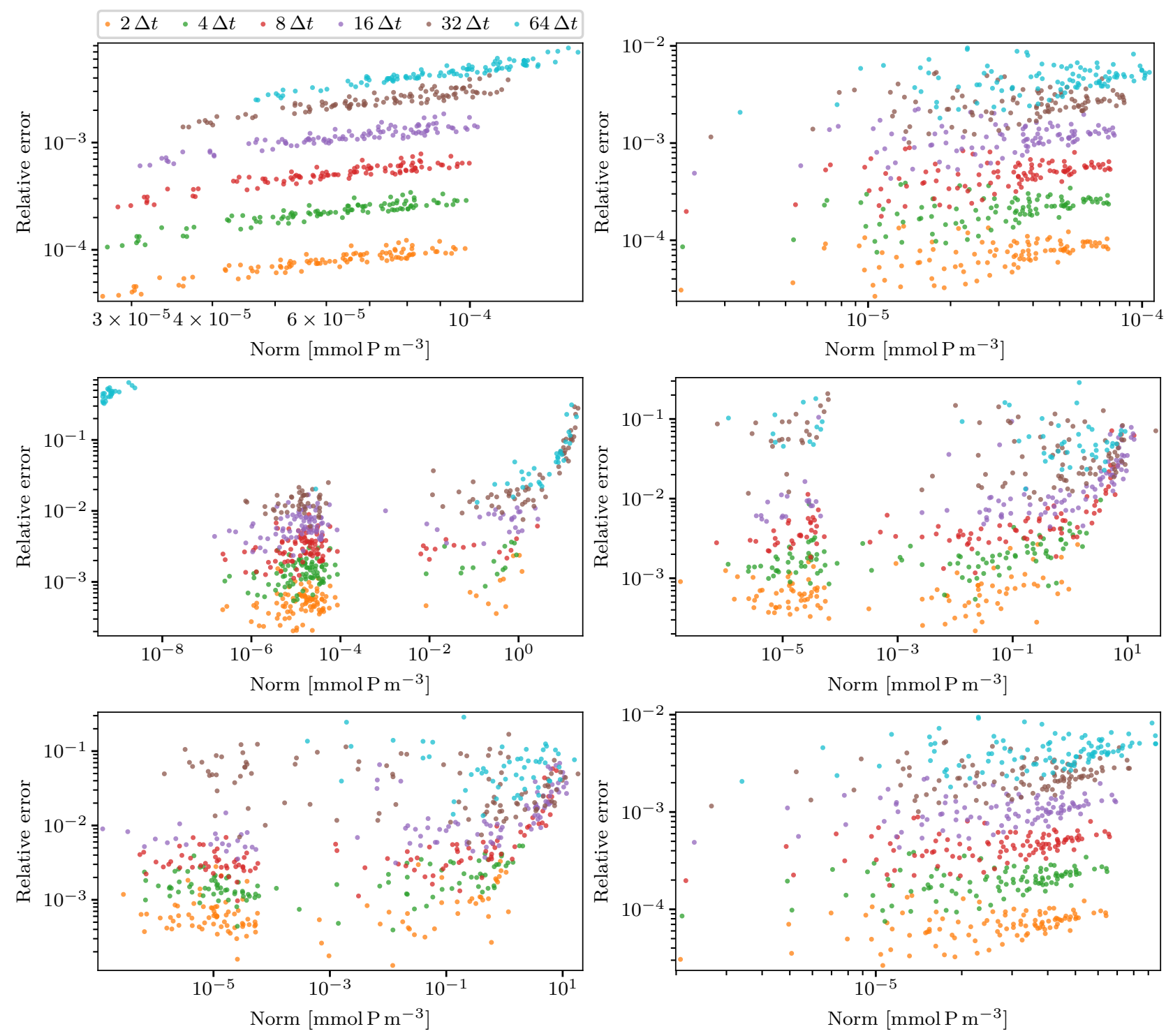

Figure 3. Norm of difference (11) and relative error (13) for $\ell=10000$ using different time steps for the spin-up. Shown are the results using a constant time step over the whole spin-up for the different biogeochemical models and all parameter vectors of the Latin hypercube sample (top left: N model, top right: N-DOP model, middle left: NP-DOP model, middle right: NPZ-DOP model, bottom left: NPZD-DOP model, bottom right: MITgcm-PO4-DOP model).

\subsubsection{Algorithm 2: Avoiding negative concentrations}

Strictly avoiding negative concentrations prevented a usage of larger time steps in most of the considered cases when calculating a steady annual cycle with the step size control. Except for the $\mathrm{N}$ model, Algorithm 2 used time step $1 \Delta t$ for the entire spin- 

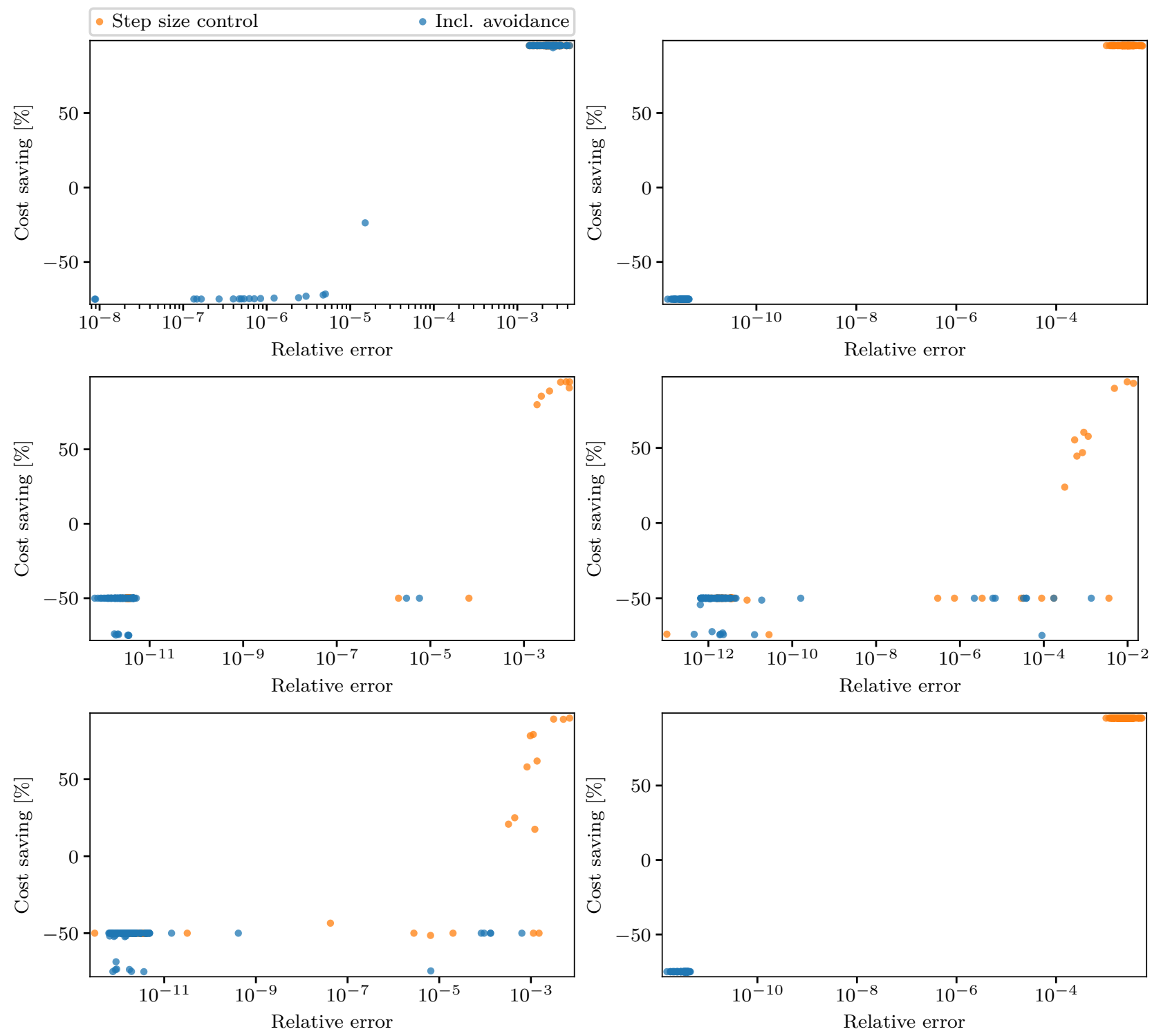

Figure 4. Saving of computational costs (14) using the step size control with the default setting and the avoidance of negative concentrations.

Shown are the results for the different biogeochemical models and all parameter vectors of the Latin hypercube sample (top left: $\mathrm{N}$ model, top right: N-DOP model, middle left: NP-DOP model, middle right: NPZ-DOP model, bottom left: NPZD-DOP model, bottom right: MITgcmPO4-DOP model). Some points of the results using the avoidance of negative concentrations obscured the points of the step size control results. For the $\mathrm{N}$ model, all points of the results using the step size control (orange points) are present in the upper right corner.

up with the reference parameter vectors of Table 2 because negative concentrations appeared for at least one tracer in all approximations calculated with larger time steps. For the $\mathrm{N}$ model, the algorithm increased the time step up the maximum at the beginning of the simulation, but accepted only time step $1 \Delta t$ after 120 model years because negative concentrations 
https://doi.org/10.5194/gmd-2021-392

Preprint. Discussion started: 7 January 2022

(c) Author(s) 2022. CC BY 4.0 License.

(c) (i)

Table 3. Relative error (13) for $\ell=10000$ obtained by the step size control algorithm (Algorithm 1) using different values of $n_{s} \in$ $\{1,2,5,10,25,50\}$.

\begin{tabular}{ccccccc}
\hline$n_{s}$ & $\mathrm{~N}$ & N-DOP & NP-DOP & NPZ-DOP & NPZD-DOP & MITgcm-PO4-DOP \\
\hline 1 & $2.258 \mathrm{e}-03$ & $2.775 \mathrm{e}-03$ & $3.439 \mathrm{e}-12$ & $1.744 \mathrm{e}-12$ & $1.845 \mathrm{e}-12$ & $2.462 \mathrm{e}-03$ \\
2 & $2.258 \mathrm{e}-03$ & $2.775 \mathrm{e}-03$ & $7.978 \mathrm{e}-13$ & $7.399 \mathrm{e}-13$ & $5.247 \mathrm{e}-13$ & $2.462 \mathrm{e}-03$ \\
5 & $2.258 \mathrm{e}-03$ & $2.775 \mathrm{e}-03$ & $5.265 \mathrm{e}-12$ & $3.068 \mathrm{e}-12$ & $2.521 \mathrm{e}-12$ & $2.462 \mathrm{e}-03$ \\
10 & $2.258 \mathrm{e}-03$ & $2.775 \mathrm{e}-03$ & $5.763 \mathrm{e}-13$ & $4.402 \mathrm{e}-13$ & $3.411 \mathrm{e}-13$ & $2.462 \mathrm{e}-03$ \\
25 & $2.258 \mathrm{e}-03$ & $2.775 \mathrm{e}-03$ & $5.756 \mathrm{e}-12$ & $3.421 \mathrm{e}-12$ & $3.565 \mathrm{e}-12$ & $2.462 \mathrm{e}-03$ \\
50 & $2.258 \mathrm{e}-03$ & $2.775 \mathrm{e}-03$ & $4.369 \mathrm{e}-12$ & $2.318 \mathrm{e}-12$ & $2.752 \mathrm{e}-12$ & $2.462 \mathrm{e}-03$ \\
\hline
\end{tabular}

arose. Accordingly, the approximation resembled the reference solution while the computational effort was huge (cost increase of 73\%, Fig. 1). For the NP-DOP, NPZ-DOP and NPZD-DOP models, the computations using the step size control with and without avoidance of negative concentrations were identical. For the N-DOP and MITgcm-PO4-DOP models, in contrast, the computational effort was quite big (an increase of about 75\%). Here, a bigger time step would be possible without considering negative values due to the error estimation. With our implementation of the step size control avoiding negative concentrations, three instead of two approximations, hence, were computed for each model year because the step size control accepted in every step the approximation calculated with the minimal time step $1 \Delta t$ (independent of negative concentrations) and increased based on the error estimation the time step for the next step. In the next step, the algorithm discarded the approximation calculated with the larger time step due to negative concentrations, and reran the approximation with the minimal time step $1 \Delta t$ again. With an adjustment of our implementation (firstly, the calculation of an approximation using the small time step to check it for negative concentrations and, secondly, the computation of the second approximation using the larger time step to estimate the local error), the computational effort could slightly be reduced to an increase to about $50 \%$.

For the Latin hypercube sample, the same qualitative results were obtained. For the $\mathrm{N}$ model, the results mostly coincided with the results of the step size control using default settings (Fig. 2). Only for a few parameter vectors, the step size control reduced the time step. This was again a result of negative concentrations during the simulation. As a consequence, the accuracy improved but at the expense of the cost saving (Fig. 4). For the other biogeochemical models, the accuracy of the approximations in Fig. 2 indicates that the Algorithm 2 computed the reference solution except for some outliers because the step size control did not increased the time step during the simulation. Thus, the computational effort was larger than for the spin-up computation of the reference solution with $1 \Delta t$ (Fig. 4).

\subsubsection{Step size control settings}

We investigated the influence of different settings for the step size control. For that purpose, we used only the reference parameter vectors listed in Table 2. 
https://doi.org/10.5194/gmd-2021-392

Preprint. Discussion started: 7 January 2022

(C) Author(s) 2022. CC BY 4.0 License.

Table 4. Relative error (13) for $\ell=10000$ obtained by the step size control algorithm (Algorithm 1) using different norms for the local error estimation.

\begin{tabular}{lcccccc}
\hline Norm & $\mathrm{N}$ & N-DOP & NP-DOP & NPZ-DOP & NPZD-DOP & MITgcm-PO4-DOP \\
\hline$\|\cdot\|_{2}$ & $2.258 \mathrm{e}-03$ & $1.214 \mathrm{e}-03$ & $3.439 \mathrm{e}-12$ & $1.744 \mathrm{e}-12$ & $1.845 \mathrm{e}-12$ & $2.462 \mathrm{e}-03$ \\
$\|\cdot\|_{2, V}$ & $2.258 \mathrm{e}-03$ & $2.775 \mathrm{e}-03$ & $3.439 \mathrm{e}-12$ & $1.744 \mathrm{e}-12$ & $1.845 \mathrm{e}-12$ & $2.462 \mathrm{e}-03$ \\
$\|\cdot\|_{2, T}$ & $2.258 \mathrm{e}-03$ & $2.775 \mathrm{e}-03$ & $5.597 \mathrm{e}-12$ & $5.537 \mathrm{e}-04$ & $4.738 \mathrm{e}-12$ & $2.462 \mathrm{e}-03$ \\
$\|\cdot\|_{2, V, T}$ & $2.258 \mathrm{e}-03$ & $2.775 \mathrm{e}-03$ & $6.213 \mathrm{e}-03$ & $1.383 \mathrm{e}-03$ & $1.398 \mathrm{e}-03$ & $2.462 \mathrm{e}-03$ \\
$\left.\|\cdot\|_{2, V}\right|_{\{1\}}$ & $1.215 \mathrm{e}-12$ & $1.885 \mathrm{e}-12$ & $1.849 \mathrm{e}-12$ & $6.879 \mathrm{e}-13$ & $1.005 \mathrm{e}-12$ & $2.195 \mathrm{e}-12$ \\
$\left.\|\cdot\|_{2, V}\right|_{\{1,2,3\}}$ & $5.021 \mathrm{e}-04$ & $1.885 \mathrm{e}-12$ & $1.849 \mathrm{e}-12$ & $6.879 \mathrm{e}-13$ & $1.005 \mathrm{e}-12$ & $2.195 \mathrm{e}-12$ \\
$\left.\|\cdot\|_{2, V}\right|_{\{11\}}$ & $2.258 \mathrm{e}-03$ & $2.775 \mathrm{e}-03$ & $1.355 \mathrm{e}-02$ & $1.804 \mathrm{e}-02$ & $1.911 \mathrm{e}-02$ & $2.462 \mathrm{e}-03$ \\
\hline
\end{tabular}

The number of model years $n_{s} \in \mathbb{N}$, after which the error estimation was computed, had no influence on the accuracy of the steady annual cycle approximation using the step size control (Table 3). For the N, N-DOP and MITgcm-PO4-DOP models, the step size control increased the time step as quickly as possible to the maximal time step regardless of the number of model years $n_{s}$ and, afterwards, applied only this maximal time step. As a consequence, the approximations for the different model years $n_{s}$ were almost identical. Similar to the results above for the NP-DOP, NPZ-DOP and NPZD-DOP models, the approximation corresponded to the reference solution for the different model years $n_{s}$ since the step size control used always time step $1 \Delta t$.

The norm used to estimate the local discretization error affected the approximation calculated with the step size control (Table 4 ) in some cases. Firstly, the application of the Euclidean norm $\|\cdot\|_{2}$ instead of the norm $\|\cdot\|_{2, V}$ influenced the step size control for the N-DOP model only. For this model, the step size control, occasionally, did not increase the time step directly to the maximal time step and finished the simulation with time step $16 \Delta t$. This led to a smaller relative error. The use of the norms $\|\cdot\|_{2, T}$ and $\|\cdot\|_{2, V, T}$, secondly, resulted in at least one increase of the time step for the NP-DOP, NPZ-DOP and NPZD-DOP models and, therefore, in a reduction of the computational costs. However, these norms had no influence for the N, N-DOP and MITgcm-PO4-DOP models. Lastly, the restriction of the norm $\|\cdot\|_{2, V}$ to different layers had an impact on the error estimation because the annual variability exclusively affects concentrations in the upper ocean, while the concentrations in the deeper ocean change very slowly. Besides, the box volumes in the upper ocean are small in comparison to the box volumes in the deeper ocean, but big boxes have a greater effect on the norm as small ones for a volume weighted norm. However, the local discretization error was high in the upper ocean due to the variability, and small in the deeper ocean. Thus, the step size control increased more frequently the step size using the restricted norms including deeper layers or including more layers (Table 4).

The choice of the initial time step especially affected the three most complex biogeochemical models. Due to the directly increasing time step up to the maximum, no differences were visible for the N, N-DOP and MITgcm-PO4-DOP models (Table 5). In contrast, the relative errors in Table 5 indicate the use of larger time steps for at least one model year for the other biogeochemical models. In particular, the step size control utilized time step $2 \Delta t$ over the entire simulation for initialization with larger time steps for the NPZ-DOP model. 
https://doi.org/10.5194/gmd-2021-392

Preprint. Discussion started: 7 January 2022

(C) Author(s) 2022. CC BY 4.0 License.

(c) (i)

Table 5. Relative error (13) for $\ell=10000$ obtained by the step size control algorithm (Algorithm 1) using different initial time steps.

\begin{tabular}{ccccccc}
\hline$m_{\text {init }}$ & $\mathrm{N}$ & N-DOP & NP-DOP & NPZ-DOP & NPZD-DOP & MITgcm-PO4-DOP \\
\hline 1 & $2.258 \mathrm{e}-03$ & $2.775 \mathrm{e}-03$ & $3.439 \mathrm{e}-12$ & $1.744 \mathrm{e}-12$ & $1.845 \mathrm{e}-12$ & $2.462 \mathrm{e}-03$ \\
$2,4,8,16,32$ & $2.258 \mathrm{e}-03$ & $2.775 \mathrm{e}-03$ & $1.848 \mathrm{e}-12$ & $5.537 \mathrm{e}-04$ & $7.119 \mathrm{e}-10$ & $2.462 \mathrm{e}-03$ \\
\hline
\end{tabular}

Table 6. Relative error (13) for $\ell=10000$ obtained by the step size control algorithm (Algorithm 1) using different tolerances $\tau_{0} \in$ $\{0.1,0.2, \ldots, 1.0\}$.

\begin{tabular}{ccccccc}
\hline$\tau_{0}$ & $\mathrm{~N}$ & N-DOP & NP-DOP & NPZ-DOP & NPZD-DOP & MITgcm-PO4-DOP \\
\hline $1.0,0.9, \ldots, 0.5$ & $2.258 \mathrm{e}-03$ & $2.775 \mathrm{e}-03$ & $3.439 \mathrm{e}-12$ & $1.744 \mathrm{e}-12$ & $1.845 \mathrm{e}-12$ & $2.462 \mathrm{e}-03$ \\
0.4 & $2.258 \mathrm{e}-03$ & $5.106 \mathrm{e}-04$ & $3.439 \mathrm{e}-12$ & $1.744 \mathrm{e}-12$ & $1.845 \mathrm{e}-12$ & $2.462 \mathrm{e}-03$ \\
0.3 & $2.258 \mathrm{e}-03$ & $3.794 \mathrm{e}-12$ & $3.439 \mathrm{e}-12$ & $1.744 \mathrm{e}-12$ & $1.845 \mathrm{e}-12$ & $4.872 \mathrm{e}-04$ \\
$0.2,0.1$ & $2.171 \mathrm{e}-12$ & $3.794 \mathrm{e}-12$ & $3.439 \mathrm{e}-12$ & $1.744 \mathrm{e}-12$ & $1.845 \mathrm{e}-12$ & $3.277 \mathrm{e}-12$ \\
\hline
\end{tabular}

The tolerance $\tau_{0} \in \mathbb{R}_{>0}$ influenced only the time step's increase for the N, N-DOP and MITgcm-PO4-DOP models. Using smaller tolerances, the possible increase to the maximal time step required more model years for the N, N-DOP and MITgcmPO4-DOP models. The use of the smallest tolerance always resulted in time step $1 \Delta t$ for the entire spin-up. For the N-DOP and MITgcm-PO4-DOP models, there existed a tolerance for which the step size control increased the time step only up to $8 \Delta t$ (Table 6). For the NP-DOP, NPZ-DOP and NPZD-DOP models, the algorithm did not increase the step when tolerance $\tau_{0}=1.0$ was used. Consequently, the choice of a smaller tolerance had no effect (Table 6).

\subsection{Algorithm 3: Decreasing time steps}

The decreasing time steps algorithm computed a reasonable approximation of the steady annual cycle with the automatic stepwise reduction of the time step and, thus, reduced the computational effort. For the $\mathrm{N}$ model with the parameter vector listed in Table 2, Fig. 5 demonstrates the similar convergence behavior towards a steady annual cycle using the decreasing time steps algorithm as well as the spin-up calculation of the reference solution. Using the decreasing time steps algorithm, the six peaks in the norm of differences (11) pertained to the six reductions of the time step. As a result of the decreased time step, a large concentration change took place in one model year similar to the large changes at the beginning of each spin-up. In particular, the reduction of the relative error resulting from the decrease of the time step is evident in Fig. 5 (right) using the tolerance 0.0001. Although the algorithm applied time step $1 \Delta t$ at the end of the spin-up computation, the spin-up did not perfectly converge against the reference solution. Nevertheless, the algorithm shortened the runtime of the simulation, and finished with an approximation of the steady annual cycle that was much better than the one of the spin-up with constant time step $64 \Delta t$, i.e., $\mathbf{y}^{10000,64}$ (cf. Fig. 3). The used tolerance affected only slightly the accuracy of the approximation, but the use of a smaller tolerance resulted in a cost saving because the decreasing time steps algorithm used each time step longer before the 


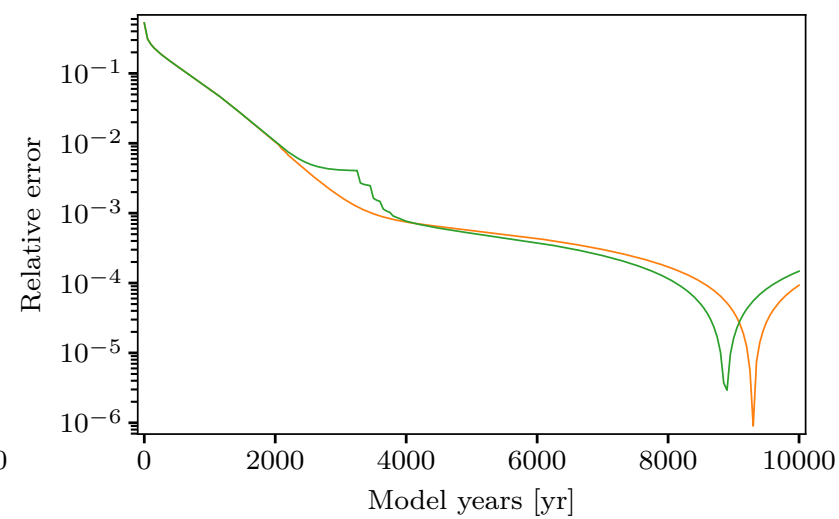

Figure 5. Results of spin-up computations for the reference parameter vector (see Table 2) and the $\mathrm{N}$ model using the decreasing time steps algorithm (Algorithm 3). Shown are the convergence of the spin-up (left), i.e., the norms of difference (11) between consecutive iterations, and the relative error (13) (right) using different tolerances $\varepsilon \in\{0.001,0.0001\}$. Furthermore, the figure of the norm of difference (left) contains the convergence towards a steady annual cycle for the reference solution. In this figure, it is mostly covered by that of the decreasing time steps algorithm with tolerance $\varepsilon=0.001$.

time step was reduced. Namely, the application of the decreasing time steps algorithm with tolerance 0.001 resulted in a cost saving of $23 \%$ compared to the spin-up computation of the reference solution and in a cost saving of $38 \%$ using the tolerance 0.0001. The results for the other biogeochemical models using the reference parameter vectors are parallel to the results shown for the $\mathrm{N}$ model.

The decreasing time steps algorithm avoided a divergent spin-up calculation but did not decrease the time step to the minimum for each simulation. We analyzed the decreasing time steps algorithm using the parameter vectors of the Latin hypcercube sample for the different biogeochemical models. For the N, N-DOP and MITgcm-PO4-DOP models, the decreasing time steps algorithm computed an appropriate approximation of the steady annual cycle for the different configurations of the tolerance $\varepsilon \in\{0.001,0.0001\}$ and the number of model years $n_{s} \in\{50,100,500\}$ as detailed in Fig. 6 (cf. Fig. 3). More specifically, the accuracy decreased slightly, on the one hand, with a smaller tolerance and, on the other hand, with a larger number of model years $n_{s}$ because in both cases larger time steps were used over a longer period of model years. Figure 7 reflected this behavior in the cost saving. The computational effort increased as a result of a more frequent check of the time step reduction or with a smaller tolerance range because the time step tended to be reduced earlier. For the NP-DOP, NPZ-DOP and NPZD-DOP models, the approximations calculated with the decreasing time steps algorithm were often identical for the different configurations. Only in half of the simulation runs decreased the algorithm the time step to $1 \Delta t$ while this occurred in more than $90 \%$ of the simulations using the other three biogeochemical models. In fact, the criterion checking the decrease of the time step was inappropriate if the norm of differences (11) oscillated (cf. Pfeil and Slawig, 2021a). The algorithm, therefore, applied the entire spin-up large time steps wherefore the relative error was high (Figs. 6 and 3). Although the algorithm depending on the configuration decreased partially the time step in these cases, the simulation using this smaller time step hardly improved the accuracy of the approximation (Fig. 7). However, the decreasing time steps algorithm automatically reduced the time step if 

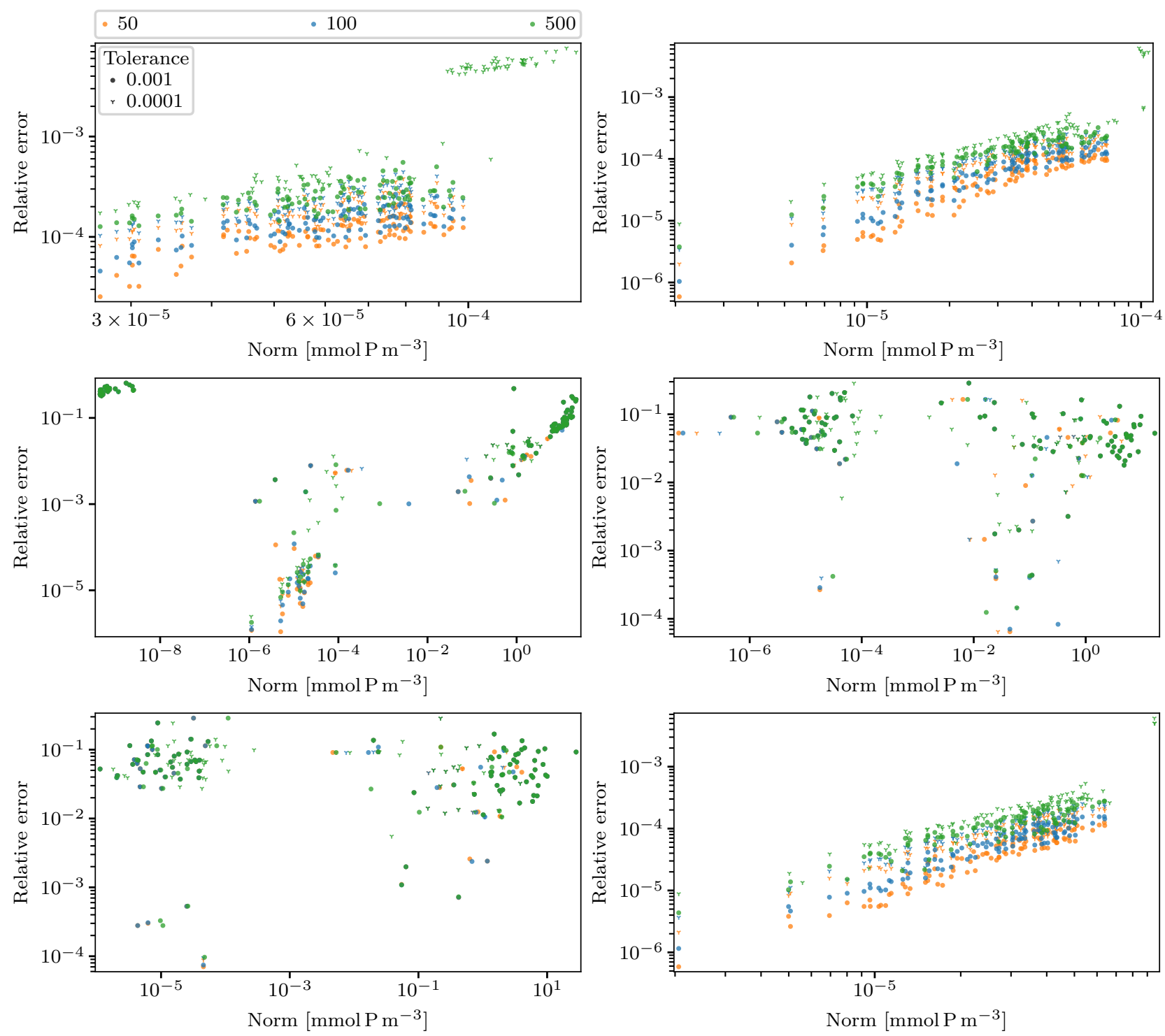

Figure 6. Norm of difference (11) and relative error (13) for $\ell=10000$ using different configurations (i.e., tolerance $\varepsilon \in\{0.001,0.0001\}$ and number of model years $\left.n_{s} \in\{50,100,500\}\right)$ of the decreasing time steps algorithm. Shown are the results for the different biogeochemical models and all parameter vectors of the Latin hypercube sample (top left: $\mathrm{N}$ model, top right: N-DOP model, middle left: NP-DOP model, middle right: NPZ-DOP model, bottom left: NPZD-DOP model, bottom right: MITgcm-PO4-DOP model).

the simulation diverged due to a too large time step. In contrast to the simulations with a low accuracy of the approximation using the NP-DOP, NPZ-DOP and NPZD-DOP models, there were also parameter vectors for which the decreasing time steps algorithm calculated an reasonable approximation with reduced computational costs (Fig. 7). 

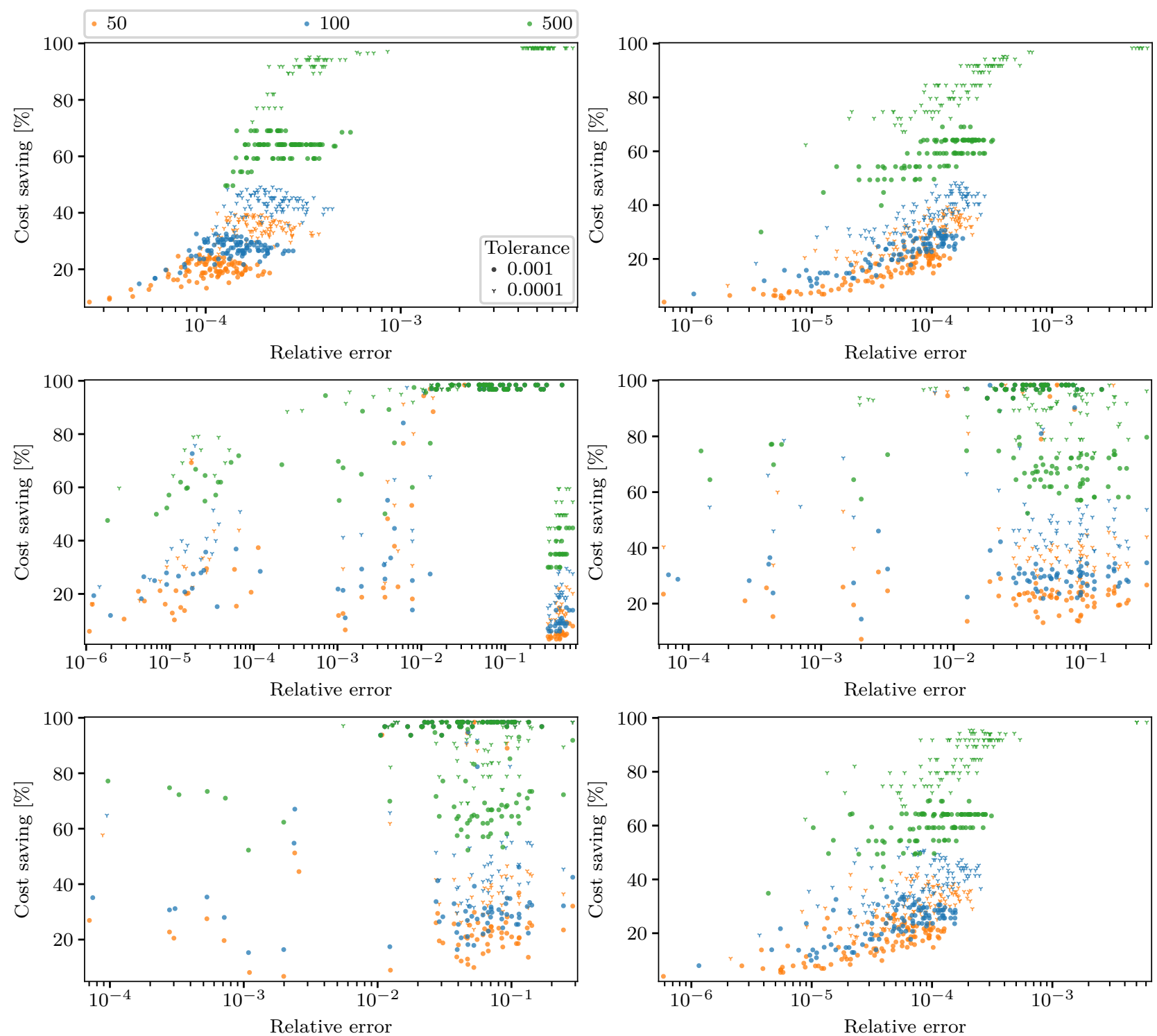

Figure 7. Saving of computational costs (14) using the decreasing time steps algorithm with different configurations (i.e., tolerance $\varepsilon \in$ $\{0.001,0.0001\}$ and number of model years $\left.n_{s} \in\{50,100,500\}\right)$. Shown are the results for the different biogeochemical models and all parameter vectors of the Latin hypercube sample (top left: $\mathrm{N}$ model, top right: N-DOP model, middle left: NP-DOP model, middle right: NPZ-DOP model, bottom left: NPZD-DOP model, bottom right: MITgcm-PO4-DOP model).

\section{Conclusions}

A reduction of the computational effort for the spin-up of marine ecosystem models is essential for the validation of these models since these computations usually require a long-time integration over several millennia (cf. Bernsen et al., 2008; Bryan, 
https://doi.org/10.5194/gmd-2021-392

Preprint. Discussion started: 7 January 2022

(c) Author(s) 2022. CC BY 4.0 License.

1984; Danabasoglu et al., 1996; Wunsch and Heimbach, 2008; Siberlin and Wunsch, 2011). Although several strategies already reduce this effort, a further reduction is desirable, such as using larger time steps based on the TMM (cf. Khatiwala et al., 2005; Khatiwala, 2007; Pfeil and Slawig, 2021a). However, the selection of an appropriate time step is a challenging compromise between accuracy and cost saving. For a classical spin-up, Pfeil and Slawig (2021a) showed the difficultly to find a bigger time step with which the spin-up provides a reasonable approximation because divergence is possible. In the current work, we introduced three algorithms that adaptively adjust the time step in the spin-up. The step size control automatically increases or decreases the time step, based on an estimate of the local discretization error (Algorithms 1 and 2). Algorithm 2 additionally avoids strictly negative tracer concentrations that might occur in the spin-up. The decreasing time steps algorithm (Algorithm 3) starts with a big time step and automatically decreases them, based on the convergence of the spin-up. In particular, these algorithms eliminated the manual selection of a suitable time step (cf. Pfeil and Slawig, 2021a).

For all three algorithms, the spin-up calculation yielded approximations of the steady annual cycle which were in exceptionally good agreement with the respective reference solution obtained with the standard time step. This holds for the considered hierarchy of six biogeochemical models and, for each model, for a reference parameter vector as well as for a Latin hypercube sample of 100 parameter vectors. As a result of an oscillating norm of differences, the computations with the decreasing time steps algorithm achieved only a low accuracy of the approximation for the most complex biogeochemical models. Additionally, the algorithms avoided divergent simulations that can occur when the (constant) time step in a classical spin-up is too large (Pfeil and Slawig, 2021a).

The automatic time step adjustment shortened the runtime of the steady annual cycle computation for many of the executed simulations. Firstly, the step size control without avoiding negative concentrations (Algorithm 1) dramatically reduced the runtime of the spin-up for some (less complex) models and the complete Latin hypercube sample of parameter vectors (cost saving of up to 95\%). For the more complex models, the algorithm, in contrast, did not achieve significant cost saving since the algorithm stayed at small time steps. In these cases, the computational effort for this algorithm, actually, was expensive because the estimation of the local error always needed two evaluations of the same time interval. Secondly, the cost saving was lost for nearly all considered configurations if negative tracer concentrations were strictly avoided as in Algorithm 2. Due to negative concentrations in the approximations, the algorithm then used nearly always the smallest time step. Therefore, the mentioned overhead for the error estimation led to a big loss in performance (cost increase of about $75 \%$ ). Thirdly, the decreasing time steps algorithm (Algorithm 3) reached the best results with respect to the performance, and lowered the computational effort up to $97 \%$. It avoided an error estimate which is why it had no significant overhead. More importantly, the computational effort of the decreasing time steps algorithm was in any case lower than for the respective reference solution. Obviously, the reduction of the computational costs is greater using a classical spin-up with a constant large time step, but the selection of a reasonable time step is difficultly (Pfeil and Slawig, 2021a).

Negative concentrations prevented a performance gain of the step size control algorithm avoiding negative concentrations (Algorithm 2). Apart from some exceptions for the $\mathrm{N}$ model, negative concentrations occurred in all simulations, especially using a time step larger than $1 \Delta t$. As a consequence, the algorithm computed the reference solution, but with a significant performance loss due to the performed estimation of the local discretization error with two approximations. However, negative 
https://doi.org/10.5194/gmd-2021-392

Preprint. Discussion started: 7 January 2022

(c) Author(s) 2022. CC BY 4.0 License.

(c) (1)

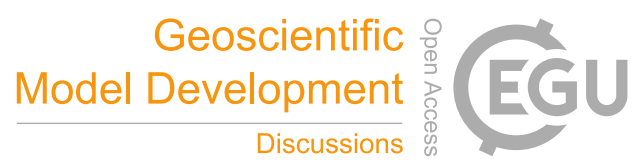

concentrations often have already occurred in the calculation of the reference solutions and of the approximations of the steady annual cycle using the other algorithms.

The advantage of the step size control algorithms and the decreasing time steps algorithm was the automatic adaptation of the time step including the selection of a reasonable time step. Although the cost saving was greater when the decreasing time steps algorithm was used compared to the step size control algorithms, the step size control algorithms, however, computed usually approximations with a higher accuracy. For the decreasing time steps algorithm, the condition to decrease the time step was in some cases unsuitable, for example for an oscillating spin-up. Future work should, therefore, include the optimization of this condition to decrease the time step in these cases or when negative concentrations occur and to improve the accuracy of the approximation. In addition, future work should analyze the occurrence of negative concentrations, especially when using the time step $1 \Delta t$.

515 In summary, the main points of this paper are the following:

- Automatic adjustment of the time step in the spin-up and, thus, automatic application of the largest possible time step without manually selecting an appropriate time step.

- Computation of an appropriate approximation of the steady annual cycle using both the step size control algorithms and the decreasing time steps algorithm.

- Reduction of the computational effort: always a reduction using the decreasing time steps algorithm (up to 97\% cost saving) and a reduction using the step size control algorithm without avoidance of negative concentrations for less complex models (up to $95 \%$ cost saving).

- No performance gain using the step size control avoiding negative concentrations due to negative concentrations in nearly all approximations.

Code and data availability. The code used to generate the data in this publication is available at https://github.com/slawig/bgc-timesteps, https://github.com/slawig/bgc-util, and https://metos3d.github.io. All used and generated data are available at https://doi.org/10.5281/zenodo. 5644003 (Pfeil and Slawig, 2021b).

Author contributions. M. Pfeil developed and implemented both algorithms and performed all simulations. M. Pfeil prepared the manuscript with contributions from T. Slawig.

Competing interests. The authors declare that they have no conflict of interest. 
https://doi.org/10.5194/gmd-2021-392

Preprint. Discussion started: 7 January 2022

(c) Author(s) 2022. CC BY 4.0 License.

(c) (i)

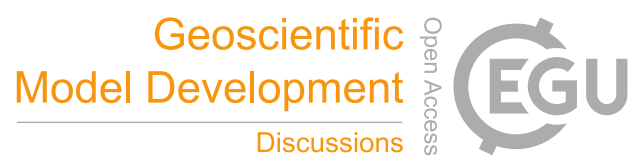

\section{References}

Bacastow, R. B. and Maier-Reimer, E.: Ocean-circulation model of the carbon cycle, Clim. Dyn., 4, 95-125, https://doi.org/10.1007/BF00208905, 1990.

Bacastow, R. B. and Maier-Reimer, E.: Dissolved organic carbon in modeling oceanic new production, Global Biogeochem. Cycles, 5, 71-85, https://doi.org/10.1029/91GB00015, 1991.

Bernsen, E., Dijkstra, H. A., and Wubs, F. W.: A method to reduce the spin-up time of ocean models, Ocean Model., 20, 380 - 392, https://doi.org/10.1016/j.ocemod.2007.10.008, 2008.

Bryan, K.: Accelerating the Convergence to Equilibrium of Ocean-Climate Models, J. Phys. Oceanogr., 14, 666-673, https://doi.org/10.1175/1520-0485(1984)014<0666:ATCTEO>2.0.CO;2, 1984.

540 Danabasoglu, G., McWilliams, J. C., and Large, W. G.: Approach to Equilibrium in Accelerated Global Oceanic Models, J. Climate, 9, 1092-1110, https://doi.org/10.1175/1520-0442(1996)009<1092:ATEIAG>2.0.CO;2, 1996.

Deuflhard, P. and Bornemann, F.: Numerische Mathematik 2, de Gruyter, Berlin, Germany, 4th revised and augmented edn., 2013.

Dutkiewicz, S., Sokolov, A. P., Scott, J., and Stone, P. H.: A three-dimensional ocean-seaice-carbon cycle model and its coupling to a twodimensional atmospheric model: Uses in climate change studies, Tech. Rep. 122, MIT Joint Program on the Science and Policy of Global Change, Cambridge, 2005.

Fasham, M. J. R., ed.: Ocean Biogeochemistry, Global Change - The IGBP Series, Springer, Berlin et al., https://doi.org/10.1007/978-3-64255844-3, 2003.

Fennel, K., Losch, M., Schröter, J., and Wenzel, M.: Testing a marine ecosystem model: sensitivity analysis and parameter optimization, J. Mar. Syst., 28, 45 - 63, https://doi.org/10.1016/S0924-7963(00)00083-X, 2001.

Khatiwala, S.: A computational framework for simulation of biogeochemical tracers in the ocean, Global Biogeochem. Cycles, 21, https://doi.org/10.1029/2007GB002923, 2007.

Khatiwala, S.: Fast spin up of Ocean biogeochemical models using matrix-free Newton-Krylov, Ocean Model., 23, 121-129, https://doi.org/10.1016/j.ocemod.2008.05.002, 2008.

Khatiwala, S., Visbeck, M., and Cane, M. A.: Accelerated simulation of passive tracers in ocean circulation models, Ocean Model., 9, 51-69, https://doi.org/10.1016/j.ocemod.2004.04.002, 2005.

Kriest, I.: Calibration of a simple and a complex model of global marine biogeochemistry, Biogeosciences, 14, 4965-4984, https://doi.org/10.5194/bg-14-4965-2017, 2017.

Kriest, I., Khatiwala, S., and Oschlies, A.: Towards an assessment of simple global marine biogeochemical models of different complexity, Prog. Oceanogr., 86, 337-360, https://doi.org/10.1016/j.pocean.2010.05.002, 2010.

Kriest, I., Sauerland, V., Khatiwala, S., Srivastav, A., and Oschlies, A.: Calibrating a global three-dimensional biogeochemical ocean model (MOPS-1.0), Geosci. Model Dev., 10, 127-154, https://doi.org/10.5194/gmd-10-127-2017, 2017.

Lee, A.: pyDOE: Design of Experiments for Python, https://pythonhosted.org/pyDOE/index.html, last access: 23 November 2021), 2014.

Marshall, J., Adcroft, A., Hill, C., Perelman, L., and Heisey, C.: A finite-volume, incompressible Navier Stokes model for studies of the ocean on parallel computers, J. Geophys. Res. Oceans, 102, 5753-5766, https://doi.org/10.1029/96JC02775, 1997.

565 Martin, J. H., Knauer, G. A., Karl, D. M., and Broenkow, W. W.: VERTEX: carbon cycling in the northeast Pacific, Deep-Sea Res. I: Oceanogr. Res. Pap., 34, 267 - 285, https://doi.org/10.1016/0198-0149(87)90086-0, 1987. 
https://doi.org/10.5194/gmd-2021-392

Preprint. Discussion started: 7 January 2022

(c) Author(s) 2022. CC BY 4.0 License.

(c) (i)

McKay, M. D., Beckman, R. J., and Conover, W. J.: A Comparison of Three Methods for Selecting Values of Input Variables in the Analysis of Output from a Computer Code, Technometrics, 21, 239-245, https://doi.org/10.2307/1268522, 1979.

Oschlies, A.: On the use of data assimilation in biogeochemical modelling, in: Ocean Weather Forecasting, edited by Chassignet, E. P. and Verron, J., pp. 525-547, Springer, Dordrecht, https://doi.org/10.1007/1-4020-4028-8_24, 2006.

Paltridge, G. W. and Platt, C. M. R.: Radiative Processes in Meteorology and Climatology, Elsevier, New York, https://doi.org/10.1002/qj.49710343713, 1976.

Parekh, P., Follows, M. J., and Boyle, E. A.: Decoupling of iron and phosphate in the global ocean, Glob. Biogeochem. Cycles, 19, https://doi.org/10.1029/2004GB002280, 2005.

575 Pfeil, M. and Slawig, T.: Shortening the runtime using larger time steps for the simulation of marine ecosystem models, arXiv [preprint], https://arxiv.org/abs/2111.09122, 17 November 2021, 2021a.

Pfeil, M. and Slawig, T.: Automatic time step adjustment for shortening the runtime of the simulation of marine ecosystem models, Zenodo [data set], https://doi.org/10.5281/zenodo.5644003, 2021b.

Piwonski, J. and Slawig, T.: Metos3D: the Marine Ecosystem Toolkit for Optimization and Simulation in 3-D - Part 1: Simulation Package v0.3.2, Geosci. Model Dev., 9, 3729-3750, https://doi.org/10.5194/gmd-9-3729-2016, 2016a.

Piwonski, J. and Slawig, T.: Temporal Coarsening of Transport Matrices with Metos3D, Tech. Rep. 1608, Department of Computer Science, Kiel University, http://www.uni-kiel.de/journals/receive/jportal_jparticle_00000291, 2016b.

Prieß, M., Piwonski, J., Koziel, S., Oschlies, A., and Slawig, T.: Accelerated parameter identification in a 3D marine biogeochemical model using surrogate-based optimization, Ocean Model., 68, 22-36, https://doi.org/10.1016/j.ocemod.2013.04.003, 2013.

585 Richardson, L. F. and Gaunt, J. A.: The deferred approach to the limit, Philos. Trans. R. Soc. A, 226, 299-361, https://doi.org/10.1098/rsta.1927.0008, 1927.

Richardson, L. F. and Glazebrook, R. T.: The approximate arithmetical solution by finite differences of physical problems involving differential equations, with an application to the stresses in a masonry dam, Philos. Trans. R. Soc. A, 210, 307-357, https://doi.org/10.1098/rsta.1911.0009, 1911.

590 Sarmiento, J. L. and Gruber, N.: Ocean biogeochemical dynamics, Princeton University Press, Princeton et al., 2006.

Schartau, M. and Oschlies, A.: Simultaneous data-based optimization of a 1d-ecosystem model at three locations in the north Atlantic: Part I - method and parameter estimates, J. Mar. Res., 61, 765-793, https://doi.org/doi:10.1357/002224003322981147, 2003.

Schmittner, A., Oschlies, A., Giraud, X., Eby, M., and Simmons, H. L.: A global model of the marine ecosystem for long-term simulations: Sensitivity to ocean mixing, buoyancy forcing, particle sinking, and dissolved organic matter cycling, Glob. Biogeochem. Cycles, 19, https://doi.org/10.1029/2004GB002283, 2005.

Siberlin, C. and Wunsch, C.: Oceanic tracer and proxy time scales revisited, Clim. Past, 7, 27-39, https://doi.org/10.5194/cp-7-27-2011, 2011.

Siewertsen, E., Piwonski, J., and Slawig, T.: Porting marine ecosystem model spin-up using transport matrices to GPUs, Geosci. Model Dev., 6, 17-28, https://doi.org/10.5194/gmd-6-17-2013, 2013.

600 Stoer, J. and Bulirsch, R.: Introduction to numerical analysis, vol. 12 of Texts in applied mathematics, Springer, New York, 3 edn., https://doi.org/10.1007/978-0-387-21738-3, 2002.

Wang, D.: A note on using the accelerated convergence method in climate models, Tellus A: Dyn. Meteorol. Oceanogr., 53, 27-34, https://doi.org/10.3402/tellusa.v53i1.12179, 2001. 
https://doi.org/10.5194/gmd-2021-392

Preprint. Discussion started: 7 January 2022

(C) Author(s) 2022. CC BY 4.0 License.

(c) (1)

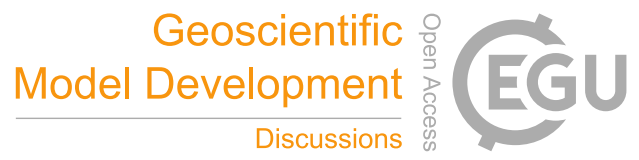

Wunsch, C. and Heimbach, P.: How long to oceanic tracer and proxy equilibrium?, Quat. Sci. Rev., 27, 637-651, https://doi.org/10.1016/j.quascirev.2008.01.006, 2008. 